\title{
A Faster Parameterized Algorithm for Treedepth*
}

Felix Reidl, Peter Rossmanith, Fernando Sánchez Villaamil, and Somnath Sikdar

Theoretical Computer Science, Department of Computer Science, RWTH Aachen University, Aachen, Germany, \{reidl, rossmani, fernando.sanchez, sikdar\}@cs.rwth-aachen.de.

August 21, 2014

\begin{abstract}
The width measure treedepth, also known as vertex ranking, centered coloring and elimination tree height, is a well-established notion which has recently seen a resurgence of interest. We present an algorithm which - given as input an $n$-vertex graph, a tree decomposition of the graph of width $w$, and an integer $t$ - decides TREedePTH, i.e. whether the treedepth of the graph is at most $t$, in time $2^{O(w t)} \cdot n$. If necessary, a witness structure for the treedepth can be constructed in the same running time. In conjunction with previous results we provide a simple algorithm and a fast algorithm which decide treedepth in time $2^{2^{O(t)}} \cdot n$ and $2^{O\left(t^{2}\right)} \cdot n$, respectively, which do not require a tree decomposition as part of their input. The former answers an open question posed by Ossona de Mendez and Nešetřil as to whether deciding TREeDEPTH admits an algorithm with a linear running time (for every fixed $t$ ) that does not rely on Courcelle's Theorem or other heavy machinery. For chordal graphs we can prove a running time of $2^{O(t \log t)} \cdot n$ for the same algorithm.
\end{abstract}

*Research funded by DFG-Project RO 927/13-1 "Pragmatic Parameterized Algorithms". 


\section{Introduction}

The notion of treedepth has been introduced several times in the literature under several different names. It seems that this was first formally studied by Pothen who used the term minimum elimination tree [25]; Katchalski et al. [17] studied the same notion under the name of ordered colorings; Bodlaender et al. in [4] used the term vertex ranking. More recently, Ossona de Mendez and Nešetril brought the same concept to the limelight in the guise of treedepth in their book Sparsity [23].

As is to be expected, there are several equivalent definitions of this term. One of the most intuitive characterizations of treedepth is via the degeneracy of the graph: a graph class has bounded treedepth if and only if the class is degenerate and there exists a constant $l$ (that depends on the class) such that no graph from the class has an induced path of length at least $l$ (Theorem 13.3 in [23]). That is, the condition that a graph has bounded treedepth imposes a slightly stronger restriction than just bounding the degeneracy of the graph: it also implies that the graph has no long induced paths. A particularly simple definition of treedepth is via the notion of vertex rankings. A $t$ ranking of a graph $G=(V, E)$ is a vertex coloring $c: V \rightarrow\{1, \ldots, t\}$ such that for any two vertices of the same color, any path connecting them has a vertex with a higher color. The minimum value of $t$ for which such a coloring exists is the treedepth or the vertex ranking number of the graph. We denote the treedepth of a graph $G$ by $\operatorname{td}(G)$. The vertex ranking number finds applications in sparse matrix factorization [13,18,20] and VLSI layout problems [19]. This notion also has important connections to the structure of sparse graphs. As Ossona de Mendez and Nešetřil show in [22], a very general class of sparse graphs, the so-called graphs of bounded expansion, can be decomposed into pieces of bounded treedepth.

Formally, the TREEDEPTH problem is to decide, given a graph $G$ and an integer $t$, whether $G$ has treedepth at most $t$. This decision problem is NPcomplete even on co-bipartite graphs as shown by Pothen [25] and later by Bodlaender et al. [4]. On trees, the problem can be decided in linear time [28]. Deogun et al. 9] showed that TREEDEPTH can be computed in polynomial time on the following graph classes: permutation, circular permutation, interval, circular-arc, trapezoid graphs and also on cocomparability graphs of bounded dimension. It is, however, NP-hard on chordal graphs [10]. On

general graphs, the problem does not admit good approximation algorithms: the best-known approximation algorithm is due to Bodlaender et al. [6] and 
has performance ratio $O\left(\log ^{2} n\right)$, where $n$ is the number of vertices in the graph. The best-known exact algorithm for this problem is due to Fomin, Giannopoulou and Pilipzcuk [15] and runs in time $O^{*}\left(1.9602^{n}\right)$. For practical applications, several simple heuristics exist. One such heuristic is to find a balanced vertex separator, assign each vertex of the separator a distinct color and then recurse on the remaining components. This method shows that $n$ vertex planar graphs have a treedepth of $O(\sqrt{n})$. There are several good heuristics for obtaining balanced separators and some of the most practically useful ones rely on spectral techniques (see, for instance [26 29]).

Concerning parameterized complexity, it is "easy" to see that TREEDEPTH is fixed-parameter tractable with the solution size as parameter. This follows from the fact that graphs of bounded treedepth are minor-closed and hence, by the celebrated Graph Minors Theorem of Robertson and Seymour, are characterized by a finite set of forbidden minors. Again, by Robertson and Seymour [27], one can test whether $H$ is a minor of a graph $G$ in time $O\left(f(h) \cdot n^{3}\right)$, where $n$ is the number of vertices in $G, h$ is the number of vertices in $H$ and $f$ is some recursive function. Therefore, for every fixed $t$, one can decide whether a graph contains as minor a member of the (finite) set that characterizes graphs of treedepth $t$ in time $O\left(g(t) \cdot n^{3}\right)$, for some recursive function $g$. In their textbook Sparsity, Ossona de Mendez and Nešetřil present in [23] an algorithm that relies on Courcelle's theorem to show that for every fixed $t$, the TREEDEPTH problem can be decided in linear time. They also present the following as an open problem:

Problem. Is there a simple linear time algorithm to check $\operatorname{td}(G) \leqslant t$ for fixed t?

Bodlaender et al. in [4] provide a dynamic programming algorithm that takes as input, a graph $G$ and a tree decomposition of $G$ of width $w$, and decides whether $G$ has treedepth at most $t$ in time $122^{O\left(w^{2} t\right)} \cdot n^{2}$. In this paper we present a linear time algorithm that decides whether $\operatorname{td}(G) \leqslant t$ in time $2^{O(w t)} \cdot n$, improving both the dependence on $w$ and $n$. If indeed $\operatorname{td}(G) \leqslant t$, then the algorithm also constructs a treedepth decomposition within this time. That a better dynamic programming algorithm can be achieved using

\footnotetext{
${ }^{1}$ We point out that the running time analysis in [4] simply states that the algorithm runs in polynomial time for a fixed $t$ and $w$. However, it is not difficult to restate the running time to include $t$ and $w$ as parameters, which is what we have done. From a personal communication [7], it seems that the running time can be improved to $2^{O\left(w^{2} t\right)} n$.
} 
treedepth leads us to believe that representing the ranking of the vertices as a tree might be algorithmically helpful in other cases.

We can then, by using previous known characteristics of treedepth, easily extend this result to get the following two algorithms:

- A simple algorithm which runs in time $2^{2^{O(t)}} \cdot n$.

- A fast algorithm which runs in time $2^{O\left(t^{2}\right)} \cdot n$ using a 5 -approximation for treewidth by Bodlaender et. al. [5].

We would like to point out that the second algorithm has a lower exponential dependency on the treedepth than the best-known algorithm to decide the treewidth of a graph, which is $2^{O\left(w^{3}\right)} \cdot n$, has on the treewidth.

\section{Preliminaries}

We use standard graph-theoretic notation (see [11] for any undefined terminology). All our graphs are finite and simple. Given a graph $G$, we use $V(G)$ and $E(G)$ to denote its vertex and edge sets. For convenience we assume that $V(G)$ is a totally ordered set, and use $u v$ instead of $\{u, v\}$ to denote the edges of $G$. For $X \subseteq V(G)$, we let $G[X]$ denote the subgraph of $G$ induced by $X$. We need the notion of edge contraction. Given an edge $e=u v$ of a graph $G$, we let $G / e$ denote the graph obtained from $G$ by contracting the edge $e$, which amounts to deleting the endpoints of $e$, introducing a new vertex $w_{u v}$, and making it adjacent to all vertices in $(N(u) \cup N(v)) \backslash\{u, v\}$. For an edge $e=u v$, by contracting $v$ into $u$, we mean contracting $e$ and renaming the vertex $w_{u v}$ by $u$. For a function $f: X \rightarrow Y$ and a set $X^{\prime} \subseteq X$ we will define applying the function on such a set to be $f\left(X^{\prime}\right)=\left\{f(x) \mid x \in X^{\prime}\right\}$.

\section{$2.1 \quad$ Forests}

We will work extensively on trees and forests. In this context, a rooted tree is a tree with a specially designated node known as the root. Let $T$ be a rooted tree with root $r$ and let $x \in V(T)$. Then an ancestor of $x$ is any node (other than itself) on the path from $r$ to $x$. Similarly a descendant of $x$ is any node (other than itself) on a path from $x$ to a leaf of $T$. In particular, $x$ is neither an ancestor nor a descendant of itself. 
A rooted forest is a disjoint union of rooted trees. Whenever we refer to a forest we will mean a rooted forest. For a node $x$ in a tree $T$ of a forest, the depth of $x$ in the forest is the number of vertices in the path from the root of $T$ to $x$ (thus the depth of the root is one). The height of a forest is the maximum depth of a node of the forest. The closure $\operatorname{clos}(F)$ of a forest $F$ is the graph with node set $\bigcup_{T \in F} V(T)$ and edge set $\{x y \mid x$ is an ancestor of $y$ in $F\}$. Furthermore we will need the notions of a subtree and the height of a node.

Definition 1 (Subtree rooted at a node). Let $x$ be a node of a tree $T$ and let $S$ be all the descendants of $x$ in $T$. The subtree of $T$ rooted at $x$, denoted by $T_{x}$, is the subtree of $T$ induced by the node set $S \cup\{x\}$ with root $x$.

Definition 2 (Subtree rooted at a node with child selection). Let $x$ be a node of a tree $T$, let $C$ be a set of children of $x$ in $T$ and let $S$ be all descendants of nodes of $C$ in $T$. The tree denoted by $T_{x}^{C}$, is the subtree of $T$ induced by the node set $S \cup C \cup\{x\}$ with root $x$.

Definition 3 (Height of a node). Let $x$ be a node of a tree $T$ and let $T_{x}$ be the subtree of $T$ rooted at $x$. Then the height of $x$ is the height of $T_{x}$.

\subsection{Treedepth and Treewidth}

Our main algorithm is a dynamic programming algorithm that works on a tree decomposition.

Definition 4 (Treewidth). Given a graph $G=(V, E)$, a tree decomposition of $G$ is an ordered pair $\left(T,\left\{W_{x} \mid x \in V(T)\right\}\right)$, where $T$ is a tree and $\left\{W_{x} \mid\right.$ $x \in V(T)\}$ is a collection of subsets of $V(G)$ such that the following hold:

1. $\cup_{x \in V(T)} W_{x}=V(G)$;

2. for every edge $e=u v$ in $G$, there exists $x \in V(T)$ such that $u, v \in W_{x}$;

3. for each vertex $u \in V(G)$, the set of nodes $x \in V(T)$ such that $u \in W_{x}$ induces a subtree of $T$.

We call the vertices of $T$ nodes (as opposed to "vertices" of $G$ ). The vertex sets $W_{x}$ are usually called bags. The width of a tree decomposition is the

size of the largest bag minus one. The treewidth of $G$, denoted $\operatorname{tw}(G)$, is the smallest width of a tree decomposition of $G$. 
In the definition above, if we restrict $T$ to being a path, we obtain the wellknown notions of a path decomposition and pathwidth. We let $\mathbf{p w}(G)$ denote the pathwidth of $G$. Let $\left(T,\left\{W_{x} \mid x \in V(T)\right\}\right)$ be a tree-decomposition; let $x \in V(T)$ and, let $S$ be the set of descendants of $x$. Then we define $V\left(\mathcal{T}_{W_{x}}\right):=\bigcup_{y \in S \cup\{x\}} W_{y}$.

We will only work on nice tree decompositions, which are tree decompositions with the following characteristics:

- Every node has either zero, one, or two children.

- Bags associated with leaf nodes contain a single vertex.

- If $x$ is a node of $T$ with a single child $x^{\prime}$ and if $X$ and $X^{\prime}$ are the bags assigned to these nodes, then either $\left|X \backslash X^{\prime}\right|=1$ or $\left|X^{\prime} \backslash X\right|=1$. In the first case, $X$ is called an introduce bag and, in the second, a forget bag.

- If $x$ is a node with two children $x_{1}$ and $x_{2}$ and if $X, X_{1}, X_{2}$ are the bags assigned to them, then $X=X_{1}=X_{2}$. We call such a bag $X$ a join bag.

Proposition $1([3])$. Given a graph $G$ with $n$ vertices and a tree decomposition of $G$ of width $w$ it is possible to compute a nice tree decomposition of $G$ of width $w$ with at most $n$ bags in linear time.

The main property of tree decompositions that we will exploit is the fact that each bag $X$ associated with an internal node is a vertex separator of $G$. Hence with each bag $X$ of a nice tree decomposition we can associate two (forget, introduce) or three (join) well-defined terminal subgraphs with terminal set $X$. For further information on treewidth and tree decompositions, we refer the reader to Bodlaender's survey [3].

A treedepth decomposition of a graph $G$ is a pair $(F, \psi)$, where $F$ is a rooted forest and $\psi: V(G) \rightarrow V(F)$ in an injective mapping such that if $u v \in E(G)$ then either $\psi(u)$ is an ancestor of $\psi(v)$ or vice versa. Whenever we deal with treedepth decompositions in this paper, the mapping $\psi$ will usually be implicit as we will have $V(G) \subseteq V(F)$.

Definition 5 (Treedepth). The treedepth $\mathbf{t d}(G)$ of a graph $G$ is the minimum height of any treedepth decomposition of $G$. 
We list some well-known facts about graphs of bounded treedepth. Proofs that are omitted can be found in [23].

1. If $\operatorname{td}(G) \leqslant d$, then $G$ has no paths with $2^{d}$ vertices and, in particular, any DFS-tree of $G$ has depth at most $2^{d}-1$.

2. If $\operatorname{td}(G) \leqslant d$, then $\mathbf{t w}(G) \leqslant \mathbf{p w}(G) \leqslant d-1$.

A useful way of thinking about graphs of bounded treedepth is that they are (sparse) graphs with no long paths.

A treedepth decomposition of a graph is not unique. One can always add extra vertices to a treedepth decomposition without increasing its height. We introduce the notion of trivially improvable treedepth decomposition so that we can differentiate between treedepth decomposition which have such unnecessary nodes and those who do not.

Definition 6 (Trivially Improvable Treedepth Decompositions). A treedepth decomposition $T$ of a graph $G$ is trivially improvable if $V(G) \subsetneq V(T)$.

We will also use extensively a special kind of treedepth decompositions that we will call nice treedepth decompositions. This notion is similar to that of minimal trees introduced in [15].

Definition 7 (Nice Treedepth Decomposition). A treedepth decomposition $T$ of $G$ is nice if the following conditions are met:

- $T$ is not trivially improvable.

- For every node $x \in V(T)$, the subgraph of $G$ induced by the nodes in $T_{x}$ is connected.

\subsection{Fixed Parameter Tractability}

Parameterized complexity deals with algorithms for decision problems whose instances consist of a pair $(x, k)$, where $k$ is a secondary measurement known as the parameter. A major goal in parameterized complexity is to investigate whether a problem with parameter $k$ admits an algorithm with running time $f(k) \cdot|x|^{O(1)}$, where $f$ is a function depending only on the parameter and $|x|$ represents the input size. Parameterized problems that admit such algorithms are called fixed-parameter tractable and the class of all such problems is denoted FPT. For an introduction to the area see [12,14,24]. 
Even if we will not explicitly mention it in the rest of the paper, it is clear that all the algorithms presented in this paper are in FPT (w.r.t. the appropriate parameter). Algorithm 1 is in FPT parameterized by the treedepth $t$ and the width $w$ of the given tree decomposition and Algorithms 3 and 4 are in FPT parameterized by the treedepth $t$.

\section{Dynamic Programming Algorithm}

In this section we present an algorithm which takes as input a triple $(G, \mathcal{T}, t)$, where $G$ is a graph $G, \mathcal{T}$ a tree decomposition of $G$ of width $w$, and $t$ an integer, and decides whether $\operatorname{td}(G) \leqslant t$ in time $2^{O(w t)} \cdot n$. For yes-instances, the algorithm can be modified to output a treedepth decomposition by backtracking. Later we will show how this algorithm can easily be used to achieve the three claimed results.

\subsection{Main Algorithm}

Our algorithm is a dynamic programming algorithm. It works by creating tables of partial decompositions. Every operation of the algorithm will take one or two sets of partial decompositions and create a new set of partial decompositions. More specifically, such an operation will be done for every bag of the tree decomposition. These partial decompositions will represent treedepth decompositions and they will have the same height as the treedepth decompositions they represent.

As such, we begin by defining partial decompositions.

Definition 8 (Partial decomposition). A partial decomposition is a triple $(F, X, h)$, where

- $F$ is a forest of rooted trees with $X \subseteq V(F)$; and,

- $h: V(F) \rightarrow \mathbb{N}^{+}$is a height function which obeys the property that for nodes $x, y \in V(F)$ with $x$ an ancestor of $y, h(x)>h(y)$.

Since we are going to use partial decompositions to represent treedepth decompositions of a graph we need to introduce a notion of height:

Definition 9 (Height of a partial decomposition). Let $(F, X, h)$ be a partial decomposition and let $R$ be the set of all roots in $F$. The height of $(F, X, h)$ is $\max _{x \in R} h(x)$. 
The following definition will be the key to keep the sets during the dynamic programming small.

Definition 10 (Partial decomposition equivalency). Two partial decompositions $\left(F_{1}, X_{1}, h_{1}\right)$ and $\left(F_{2}, X_{2}, h_{2}\right)$ are equivalent if and only if $X_{1}=X_{2}$ and there exists a bijective function $\psi: V\left(F_{1}\right) \rightarrow V\left(F_{2}\right)$ such that the following hold:

- the function $\psi$ is an isomorphism between $F_{1}$ and $F_{2}$;

- for all $x \in X_{1}, \psi(x)=x$, that is, $\psi$ is the identity map when restricted to the set $X_{1}$;

- for every node $v$ in the forest $F_{1}, h_{1}(v)=h_{2}(\psi(v))$.

Clearly two equivalent partial decompositions have the same height. Later we will show that it suffices to keep a representative for each equivalency class during the dynamic programming. We will do this by proving that we only care about a specific restricted type of treedepth decompositions and showing that we have a partial decomposition representing everyone of these important treedepth decompositions. Since their height are the same, we can read the heights of all important treedepth decompositions from the heights of the partial decompositions representing them.

It should be noted that the algorithm is oblivious to the fact that there is a connection between partial decompositions and treedepth decompositions, i.e. the properties that connect partial decompositions with treedepth decompositions are only implicit. This works because we can disregard every part a treedepth decompositions that does not contain a node of the bag we are currently working on.

The following definition will be helpful to decrease the size of the tables when the size of the bags decreases. The way in which we will connect treedepth decompositions to partial decompositions will be based on this operation.

Definition 11 (Restriction of a partial decomposition). The restriction of a partial decomposition $(F, X, h)$ to $X^{\prime} \subseteq X$ is the partial decomposition $\left(F^{\prime}, X^{\prime}, h^{\prime}\right)$, where $F^{\prime}$ is obtained by iteratively deleting the leaves of the forest $F$ that are not in $X^{\prime}$. The height function $h^{\prime}$ is obtained from $h$ by restricting it to $V\left(F^{\prime}\right)$. 
As we move from the leaves to the root of the tree decomposition we will need a relationship between the previous set of partial decompositions and the new ones we want to compute. The most important thing that we have to make sure of is that the predecessor relationship is maintained, since this is what permits there to be an edge in a treedepth decomposition. The following definitions will be used to make sure that the predecessor relation is kept intact.

Definition 12 (Topological generalization). Let $F_{1}, F_{2}$ be rooted forests and let $X$ be a set of vertices such that $X \subseteq V\left(F_{1}\right) \cap V\left(F_{2}\right)$. We say $F_{1}$ topologically generalizes $F_{2}$ under $X$ if there exists an injective mapping $f: V\left(F_{2}\right) \rightarrow V\left(F_{1}\right)$ where the following conditions hold:

- $\left.f\right|_{X}=i d$.

- For any node $x \in V\left(F_{2}\right)$ and an ancestor $y$ of $x, f(y)$ is an ancestor of $f(x)$ in $F_{1}$

We say that a partial decomposition $\left(F_{1}, X_{1}, h_{1}\right)$ topologically generalizes a partial decomposition $\left(F_{2}, X_{2}, h_{2}\right)$ if $X_{2} \subseteq X_{1}$ and $F_{1}$ topologically generalizes $F_{2}$ under $X_{2}$.

We will also show that it suffices to work on rooted graphs. This is not fundamental to the algorithm, but it will make its description and proof of correctness much easier.

Definition 13 (Rooted graph). A rooted graph $G=(V, E, r)$ is a graph with the specified universal vertex $r \in V(G)$ which is connected to every other vertex of $G$.

These definitions are all that are needed to describe the algorithm. We next define the operations we will perform on the bags of a tree decomposition of a rooted graph $G$. We try to describe these operations in a succinct manner. It is not immediately obvious why these operations should provide the previously described full characterization of all relevant treedepth decompositions. This will be fully explained in the next subsection.

During the forget operation we restrict every partial decomposition that we have on a smaller set and only keep one representative for every equivalency class. This will make our tables smaller. 
Definition 14 (Forgetting a vertex from a partial decomposition). Let $G$ be a graph, let $X \subseteq V(G)$ and let $R^{\prime}$ be a set of partial decompositions on the set $X$. For a vertex $u \in X$, the forget operation on $u$ denoted by forget $\left(R^{\prime}, X, u\right)$ is defined to be a set $A$ of partial decompositions obtained as follows: initially set $A \leftarrow \varnothing$; for every partial decomposition $\left(F^{\prime}, X^{\prime}, h^{\prime}\right) \in R^{\prime}$, consider its restriction to the set $X \backslash\{u\}$ and add it to the set $A$ only if it is not equivalent to any member in $A$.

Note that the set $A$ is not unique and that it contains only non-equivalent partial decompositions obtained by restricting the decompositions in $R^{\prime}$ to $X \backslash\{u\}$.

The introduce operation is somewhat more involved. Given a set $R^{\prime}$ of partial decompositions of the form $\left(F^{\prime}, X^{\prime}, h^{\prime}\right)$ where $X^{\prime} \subseteq V(G)$, the result of introducing $u \in V(G) \backslash X^{\prime}$ is a set $A$ of partial decompositions whose elements $(F, X, h)$ are computed as follows:

1. Guess every forest $F$ which complies with certain conditions.

2. Find a partial decomposition $\left(F^{\prime}, X^{\prime}, h^{\prime}\right) \in R^{\prime}$ such that $F$ topologically generalizes $F^{\prime}$. If no such partial decomposition exists, discard $F$.

3. Given $F$ and $F^{\prime}$, for every function $f$ that witnesses $F$ topologically generalizing $F^{\prime}$, create a partial decomposition of the form $(F, X=$ $\left.X^{\prime} \cup\{u\}, h\right)$.

4. Add $(F, X, h)$ to $A$ if its height is smaller than $t$ and there is no equivalent partial decomposition already contained in $A$.

Formally this translates to the following:

Definition 15 (Vertex introduction into a partial decomposition). Let $G=$ $(V, E, r)$ be a rooted graph, let $X^{\prime} \subseteq V(G)$ and let $R^{\prime}$ be a set of partial decompositions of the form $\left(F^{\prime}, X^{\prime}, h^{\prime}\right)$. For a vertex $u \in V(G) \backslash X^{\prime}$ and an integer $t$, the introduction operation on $u$ denoted by $\operatorname{intro}_{t}\left(R^{\prime}, X^{\prime}, u, G\right)$ is defined to be a set $A$ of partial decompositions constructed as follows:

Let $X=X^{\prime} \cup\{u\}$. Initialize $S \leftarrow \varnothing$. Generate every tree $F$ with up to $|X| \cdot t$ vertices which fulfills the following properties:

- $r$ is the root of $F$.

- $X \subseteq V(F)$. 
- All leafs of $F$ are in $X$.

- $E(G[X]) \subseteq E(\operatorname{clos}(F)[X])$.

For every partial decomposition $\left(F^{\prime}, X^{\prime}, h^{\prime}\right) \in R^{\prime}$ and every function $f: V\left(F^{\prime}\right) \rightarrow V(F)$ that witnesses that $F$ topologically generalizes $F^{\prime}$ on the set $X \backslash\{u\}$ add $\left(F,\left(F^{\prime}, X^{\prime}, h^{\prime}\right), f\right)$ to $S$ if $f\left(F^{\prime}\right)=V(F) \backslash\{u\}$.

For every $\left(F,\left(F^{\prime}, X^{\prime}, h^{\prime}\right), f\right) \in S$ compute the partial decomposition $(F, X, h)$, where $h$ is defined recursively by visiting the vertices of $F$ in depth-first postorder fashion. Let $z \in F$ and let $C$ be the set of children of $z$ in $F$. When $z$ is visited, if $z \neq u$ and there exists a node $z^{\prime} \in V\left(F^{\prime}\right)$ such that $f\left(z^{\prime}\right)=z$, set $h(z)=\max \left\{\max _{c \in C} h(c)+1, h^{\prime}\left(z^{\prime}\right)\right\}$. Else for any other node $z \in V(F)$ set $h(z)=\max _{c \in C} h(c)+12$ Finally add the partial decomposition $(F, X, h)$ to the set $A$, if its height is smaller that $t$ and $A$ does not contain an equivalent partial decomposition to $(F, X, h)$.

Definition 16 (Joining Partial Decompositions). Let $G=(V, E, r)$ be a rooted graph. Let $R_{1}$ and $R_{2}$ be two sets of partial decompositions on $X \subseteq$

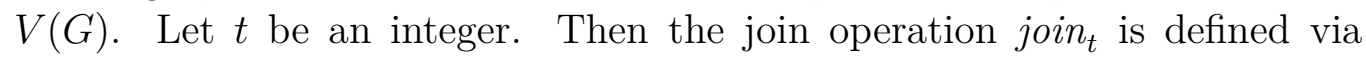
$\operatorname{join}_{t}\left(X, R_{1}, R_{2}, G\right)=A$, where $A$ is a set of partial decompositions which is constructed as follows:

Initialize $S \leftarrow \varnothing$. Generate every tree $F$ with up to $|X| \cdot t$ vertices which fulfills the following properties:

- $r$ is the root of $F$.

- $X \subseteq V(F)$.

- All leaves of $F$ are in $X$.

Take every pair of partial decompositions $\left(F_{1}, X, h_{1}\right) \in R_{1}$ and $\left(F_{2}, X, h_{2}\right) \in$ $R_{2}$ and every pair of functions $f_{1}$ and $f_{2}$ which witness that $F$ topologically generalizes $F_{1}$ and $F_{2}$ on the set $X$ respectively. Add the tuple $\left(F,\left(F_{1}, X, h_{1}\right),\left(F_{2}, X, h_{2}\right), f_{1}, f_{2}\right)$ to $S$ if $f_{1}\left(F_{1}\right) \cap f_{2}\left(F_{2}\right)=X$ and $f_{1}\left(F_{1}\right) \cup f_{2}\left(F_{2}\right)=V(F)$.

For every $\left(F,\left(F_{1}, X, h_{1}\right),\left(F_{2}, X, h_{2}\right), f_{1}, f_{2}\right) \in S$ we get one partial decomposition $(F, X, h)$ where $h$ is defined as follows: The function $h$ is defined recursively by visiting the vertices of $F$ in depth-first post-order fashion. Let $z \in F$ and let $C$ be the set of children of $z$ in $F$. Let $\alpha_{1}=h_{1}\left(z_{1}\right)$ if there exists $z_{1}$ such that $f_{1}\left(z_{1}\right)=z$ and $\alpha_{1}=1$ otherwise. Analogously, let $\alpha_{2}=h_{2}\left(z_{2}\right)$ if

\footnotetext{
${ }^{2}$ We define the maximum over the empty set to be zero.
} 
there exists $z_{2}$ such that $f_{2}\left(z_{2}\right)=z$ and $\alpha_{2}=1$ otherwise. Then we compute the height of $z$ as $h(z)=\max \left\{\max _{c \in C} h(c)+1, \alpha_{1}, \alpha_{2}\right\}$.

Finally add the partial decomposition $(F, X, h)$ to the set $A$, if its height is smaller that $t$ and $A$ does not contain an equivalent partial decomposition to $(F, X, h)$.

The main algorithm can be found in Algorithm 1. We claim that this algorithm correctly decides, given an $n$-vertex graph $G$ and a tree decompo-

sition of width at most $w$, whether $G$ has treedepth at most $t$ in time $2^{O(w t)} \cdot n$. The rest of this section is dedicated to proving this statement.

\subsection{Correctness of Algorithm 1}

To prove the correctness of this algorithm we will show a relationship between treedepth decompositions and restrictions. Our proof can be divided in the following steps:

1. We show that every graph $G$ admits a nice treedepth decomposition of height $\operatorname{td}(G)$ (Lemma 3).

2. We show that it is sufficient to work with rooted graphs and that such graphs have an optimal nice treedepth decomposition $T$ such that root of $T$ is the root of graph (Lemma 9).

3. We define the restriction of a tree. Since in this context we treat tree decompositions as trees, this will provide a relationship between treedepth decompositions and partial decompositions (Definition 17).

4. We use the previous facts to show that for any nice treedepth decomposition of the graph, our table contains its restriction (Lemma 12);

5. and moreover every partial decomposition contained in the table is a restriction of some treedepth decomposition of the graph (Lemma 13).

All this together achieves the desired result.

We will prefer to work with decompositions that are not trivially improvable. The next lemma shows that one can always obtain such a decomposition from a trivially improvable one without increasing the height. 

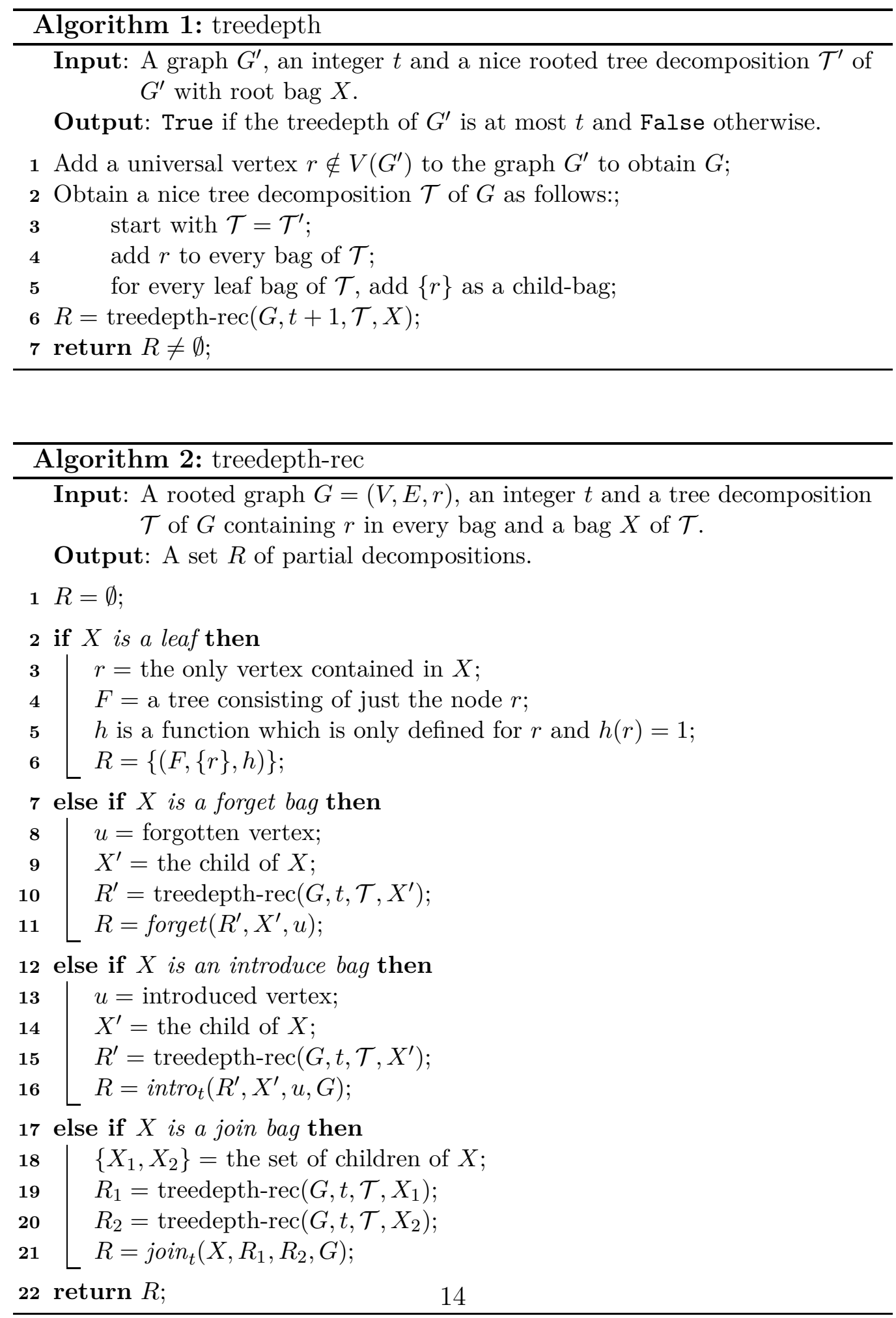
Lemma 1. Let $T$ be a trivially improvable treedepth decomposition of a graph $G$ of height h. Let $x \in V(T) \backslash V(G)$ be a root of some tree in the decomposition $T$. Then the decomposition obtained by removing $x$ is a treedepth decomposition of $G$ with height at most $h$.

Proof. Since $x \notin V(G)$, we have that $G \subseteq \operatorname{clos}(T \backslash\{x\})$. Thus $T \backslash\{x\}$ is a treedepth decomposition of $G$. Clearly the height does not increase on deleting $x$.

Lemma 2. Let $T$ be a trivially improvable treedepth decomposition of a graph $G$ with height $h$. Suppose that $x \in V(T) \backslash V(G)$ be a non-root node and let $y$ be its parent in $T$. Then the treedepth decomposition obtained by contracting the edge $x y$ is a treedepth decomposition of $G$ with height at most $h$.

Proof. Suppose $T^{\prime}$ is the forest obtained by contracting the edge $x y$. Then the height of $T^{\prime}$ is at most $h$. If $a, b \in V(T)$ is an ancestor-descendant pair that represents an edge of $G$, then these vertices form an ancestor-descendant pair in $T^{\prime}$ too. Thus $T^{\prime}$ is a treedepth decomposition of $G$ with height at most $h$.

Corollary 1. Given a trivially improvable treedepth decomposition $T$ of a graph $G$, one can obtain a decomposition of $G$ that is not trivially improvable in time polynomial in $|T|$.

Proof. Apply either Lemma 1 or 2 until $V(T)=V(G)$.

The operations described in Lemma 1 and Lemma 2 does not increase the height of a decomposition. It therefore suffices to work with decompositions that are not trivially improvable. We will now use these results to proof certain properties of nice treedepth decomposition. In a sense, nice treedepth decompositions are those whose structure cannot be easily improved.

Lemma 3. Every graph $G$ admits a nice treedepth decomposition of height $\operatorname{td}(G)$.

Proof. Let us assume $G$ to be connected. If $G$ has more than one connected component then we can apply this argument to each component in turn.

By Corollary 1, it is sufficient to show that given an optimal treedepth decomposition that is not trivially improvable, one can construct a decomposition of the same height that is nice. Therefore let $T$ be an optimal decomposition of $G$ with root $r$ that is not trivially improvable and let $x \in V(T)$ 
be a node at which the niceness condition is violated. That is, the subgraph $G\left[V\left(T_{x}\right)\right]$ of $G$ induced by the vertices in the subtree of $T$ rooted at $x$ has as connected components $C_{1}, \ldots, C_{l}$, where $l \geqslant 2$, and suppose that $x \in V\left(C_{1}\right)$. Note that $T_{x}$ itself is a trivially improvable decomposition of $C_{i}$ for each $1 \leqslant i \leqslant l$. Repeatedly use Lemma 1 to obtain a decomposition $T_{i}$ for $C_{i}$ which is not trivially improvable such that height $\left(T_{i}\right) \leqslant \operatorname{height}\left(T_{x}\right)$. Note that the root of $T_{1}$ is $x$.

If $x^{\prime}$ is the parent of $x$ in $T$, then for every component $C_{i}$, there exists at least one node $y$ in the $\left(r, x^{\prime}\right)$-path in $T$ such that $y$ has an edge to $C_{i}$ in the graph $G$. This follows because $G$ is connected and the only vertices that $C_{i}$ can be connected to are on the $\left(r, x^{\prime}\right)$-path in $T$. Thus for each component $C_{i}$, we can identify a node $y_{i}$ on the $\left(r, x^{\prime}\right)$-path such that $y_{i}$ has the maximum distance from $r$ among all vertices on the path that are connected to $C_{i}$ in the graph $G$. Construct a new tree $T^{\prime}$ from $T$ by deleting $T_{x}$ and, for $1 \leqslant i \leqslant l$, attaching $\operatorname{root}\left(T_{i}\right)$ to $y_{i}$. We claim that $T^{\prime}$ is a treedepth decomposition of $G$ of height at most height $(T)$; that the subgraph of $G$ induced by the vertices in the subtree $T_{x}^{\prime}$ is connected; and, that if this construction is repeated on the tree $T^{\prime}$ to obtain a tree $T^{\prime \prime}$, then $T_{x}^{\prime \prime}$ induces a connected subgraph of $G$.

That $T^{\prime}$ is a treedepth decomposition of $G$ is easy to see as we connected $T_{i}$ to the "deepest node" on the $\left(r, x^{\prime}\right)$-path that has an edge to $C_{i}$. Consequently, all neighbors of $C_{i}$ on the $\left(r, x^{\prime}\right)$-path are ancestors of the root of $T_{i}$ in $T^{\prime}$. We therefore have $G \subseteq \operatorname{clos}\left(T^{\prime}\right)$. The height of $T^{\prime}$ cannot increase since each $T_{i}$ has height at most height $\left(T_{x}\right)$ and they are connected to vertices on the $\left(r, x^{\prime}\right)$-path (which are "above" the node $\left.x\right)$. What is also clear is that $T_{x}^{\prime}=T_{1}$ induces a connected subgraph of $G$. Suppose that this procedure is repeated on a node $z \in V\left(T^{\prime}\right)$ to obtain $T^{\prime \prime}$.

We distinguish two cases. First suppose that $z \notin V\left(T_{x}^{\prime}\right)$. To construct $T^{\prime \prime}$, one would delete $T_{z}^{\prime}$ from $T^{\prime}$ and add trees $T_{1}^{\prime}, \ldots, T_{p}^{\prime}$ to vertices on the $\left(r, z^{\prime}\right)$ path in $T^{\prime}$, where $z^{\prime}$ is the parent of $z$ in $T^{\prime}$. The crucial observation here is that since $z \notin V\left(T_{x}^{\prime}\right), x$ does not appear in the $\left(r, z^{\prime}\right)$-path. For if $x$ did appear on this path, we would have had $z \in V\left(T_{x}^{\prime}\right)$, contradicting our assumption that this is not the case. Note that it might be that $T_{x}^{\prime}$ is a subtree of $T_{z}^{\prime}$ and therefore one of the connected components, say $C_{1}^{\prime}$, of $G\left[V\left(T_{z}^{\prime}\right)\right]$ contains the vertices of $T_{x}^{\prime}$ (and perhaps more). Construct a treedepth decomposition of $C_{1}^{\prime}$ whic is not trivially improvable by starting out with $T_{z}^{\prime}$ and using Lemma 1 to remove redundant vertices. Call this decomposition $T_{1}^{\prime}$ and observe that $T_{x}^{\prime}$ is a subtree of it. Construct decompositions $T_{2}^{\prime}, \ldots, T_{p}^{\prime}$ for the remaining components $C_{2}^{\prime}, \ldots, C_{p}^{\prime}$ of $G\left[T_{z}^{\prime}\right]$. Then, as before, add trees $T_{i}^{\prime}$ to the deepest 
node on the $\left(r, z^{\prime}\right)$-path that has an edge to $C_{i}^{\prime}$. Since this did not modify $T_{x}^{\prime}$, we have $T_{x}^{\prime \prime}=T_{x}^{\prime}$.

Next suppose that $z \in V\left(T_{x}^{\prime}\right)$. Then $z \neq x$, since the subgraph of $G$ induced by $T_{x}^{\prime}$ is connected. Thus $z$ must lie "deeper" in the tree $T_{x}^{\prime}$. Let $z^{\prime}$ be the parent of $z$ in $T^{\prime}\left(z^{\prime}\right.$ may be $\left.x\right)$. Suppose that $T^{\prime}$ is modified by deleting $T_{z}^{\prime}$ and adding the trees $T_{1}^{\prime}, \ldots, T_{p}^{\prime}$ to vertices $z_{1}, \ldots, z_{p}$ on the $\left(r, z^{\prime}\right)$-path. Recall that that $z_{i}$ is the "deepest node" on the $\left(r, z^{\prime}\right)$-path to which $T_{i}^{\prime}$ has an edge (when viewed as vertices of $G$ ). We claim that each $z_{i}$ is a descendant of $x$. Had this not been the case then some $z_{i}$ would be an ancestor of $x$ in $T^{\prime}$, and the subgraph of $G$ induced by $T_{x}^{\prime}$ would not have been connected. What this shows is that once a node has been handled, it no longer has to be handled again. Therefore by repeating this procedure at most $|V(G)|$ many times, we can obtain a treedepth decomposition which is nice and of height at most height $(T)$. Since the time taken to effect this transformation per node is polynomial in $|G|$, the overall time taken to transform a decomposition which is not trivially improvable to a nice decomposition is also polynomial in $|G|$.

As a result of Corollary 1 and the proof of Lemma 3, we obtain the following easy-to-prove, yet, important result.

Corollary 2. Let $T$ be a treedepth decomposition of a graph $G$. One can compute in time polynomial in $|G|$, a nice treedepth decomposition $T^{\prime}$ with the following properties:

1. height $\left(T^{\prime}\right) \leqslant \operatorname{height}(T)$;

2. for each vertex $x \in V(G)$, height $t_{T^{\prime}}(x) \leqslant \operatorname{height}_{T}(x)$;

3. for any node $x \in V\left(T^{\prime}\right)$, we have that $A n c_{T^{\prime}}(x) \subseteq A n c_{T}(x)$ and $\operatorname{Des}_{T^{\prime}}(x) \subseteq$ $\operatorname{Des}_{T}(x)$.

Given that one can transform any treedepth decomposition $T$ into one that is nice and not trivially improvable in time polynomial in $|V(T)|$, we will henceforth assume that the treedepth decompositions that we deal with have this property. Here is another property of treedepth decompositions that will prove to be useful to us later.

Lemma 4. Let $T$ be a nice treedepth decomposition of a graph $G$. Let $x \in$ $V(G)$ be a vertex such that $x$ is not a leaf in $T$. If $y$ is a child of $x$ in $T$, then there exists an edge $x c \in E(G)$, for some $c \in V\left(T_{y}\right)$. 
Proof. Since $T$ is a nice treedepth decomposition, the subtree $T_{x}$ rooted at $x$ induces a connected subgraph of $G$. From the definition of a treedepth decomposition, it follows that there can be no edge in $G$ adjacent to a node of $V\left(T_{y}\right)$ and a node of $\left(V\left(T_{x}\right) \backslash\{x\}\right) \backslash V\left(T_{y}\right)$. From this it follows that for $G\left[V\left(T_{x}\right)\right]$ to be connected, there must be an edge between $x$ and some node of $T_{y}$.

Thus every inner node in a nice treedepth decomposition has an edge to at least one of its descendants (in the graph represented by the decomposition).

Lemma 5. Given a nice treedepth decomposition $T$ of a graph $G$, let $x \in$ $V(G)$ and let $C=$ Children $_{T}(x)$. For $C^{\prime} \subseteq C$, let $T_{x}^{C^{\prime}}$ denote the tree obtained from $T_{x}$ by deleting the subtrees rooted at the vertices of $C \backslash C^{\prime}$. Then $G\left[V\left(T_{x}^{C^{\prime}}\right)\right]$ is a connected subgraph of $G$.

Proof. Since $T$ is a nice treedepth decomposition, it follows that for every $c \in C$ the subtree $T_{c}$ of $T$ rooted at $c$ induces a connected subgraph of $G$. From Lemma 4, it follows that $x$ is connected to a node of $T_{c}$. Thus the lemma follows.

As mentioned before, our algorithm works on partial decompositions. Let us first define a relation between treedepth decompositions and partial decompositions by defining what we call the restriction of a tree. Remember that we treat treedepth decompositions as trees, and we will do so often.

Definition 17 (Restriction of a tree ). Given a tree $T$, let $(T, V(T), h)$ be the partial decomposition where $h(x)=$ height $_{T}(x)$ for all $x \in V(T)$. For $X \subseteq V(T)$, let $(F, X, h)$ be the restriction of $(T, V(T), h)$ to the set $X$. A partial decomposition $\left(F^{\prime}, X, h^{\prime}\right)$ is a restriction of $T$ if $\left(F^{\prime}, X, h^{\prime}\right)$ is equivalent to $(F, X, h)$. We call the function $\psi: V\left(F^{\prime}\right) \rightarrow V(F)$ that witnesses the equivalency as per Definition 10 of these two restrictions the witness of the restriction.

The following properties of restrictions will prove to be useful later on.

Lemma 6. Let $(F, X, h)$ be a partial decomposition. For $X^{\prime} \subseteq X$, let $\left(F^{\prime}, X^{\prime}, h^{\prime}\right)$ be the restriction of $(F, X, h)$ to $X^{\prime}$. Then for any $X^{\prime \prime} \subseteq X^{\prime}$, the restrictions of $\left(F^{\prime}, X^{\prime}, h^{\prime}\right)$ and $(F, X, h)$ to $X^{\prime \prime}$ are identical. 
Proof. First observe that if $x$ is a leaf in $F$ then for any $y \neq x, x$ is a leaf in $F-y$. Moreover if we restrict the decomposition $(F, X, h)$ to $X^{\prime \prime}$, then the only leaves of the forest are elements of $X^{\prime \prime}$. Suppose that the restrictions of $\left(F^{\prime}, X^{\prime}, h^{\prime}\right)$ and $(F, X, h)$ to $X^{\prime \prime}$ yields (respectively) the decompositions $\left(\widetilde{F}^{\prime}, X^{\prime \prime}, \widetilde{h}^{\prime}\right)$ and $\left(\widetilde{F}, X^{\prime \prime}, \widetilde{h}\right)$. Let $s_{1}=v_{1}, \ldots, v_{p}$ be the sequence in which vertices were deleted to obtain $\left(\widetilde{F}^{\prime}, X^{\prime \prime}, \widetilde{h}^{\prime}\right)$ from $(F, X, h)$; and, $s_{2}=w_{1}, \ldots, w_{q}$ were the vertices that were deleted to obtain $\left(\widetilde{F}, X^{\prime \prime}, \widetilde{h}\right)$ from $(F, X, h)$.

Suppose that there exists a node $y$ in the sequence $s_{1}$ that does not occur in $s_{2}$ and suppose that $v$ is the first such node of $s_{1}$ so that $s_{1}=$ $v_{1}, \ldots, v_{l}, v, \ldots$. Note that $v$ is a leaf after the vertices $v_{1}, \ldots, v_{l}$ are deleted, irrespective of the order of deletion. Since the vertices $v_{1}, \ldots, v_{l}$ occur in $s_{2}$, suppose that $w_{i}$ is the last of these that occur in $s_{2}$. Then after the deletion of $w_{i}$ (in the sequence $s_{2}$ ), the node $v$ continues to remain as a leaf and this fact does not change with further deletions down the sequence $s_{2}$. But $v$ was deleted in the sequence $s_{1}$ and hence $v \notin X^{\prime \prime}$ and the fact that $v$ does not appear in the sequence $s_{2}$ implies that $\widetilde{F}$ has a leaf node that is not an element of $X^{\prime \prime}$, a contradiction. This shows that every node of $s_{1}$ appears in $s_{2}$. Reversing the argument, one sees that every node in $s_{2}$ appears in $s_{1}$. Hence $s_{1}$ and $s_{2}$ contain the same vertices, possibly in a different order. Therefore $V(\widetilde{F})=V\left(\widetilde{F}^{\prime}\right)$ and the partial decompositions $\left(\widetilde{F}^{\prime}, X^{\prime \prime}, \widetilde{h}^{\prime}\right)$ and $\left(\widetilde{F}, X^{\prime \prime}, \widetilde{h}\right)$ are identical.

Lemma 6 immediately implies the following:

Corollary 3. Let $(F, X, h)$ be a partial decomposition and let $X^{\prime} \subseteq X$. The restriction of $(F, X, h)$ on $X^{\prime}$ is unique up to isomorphism.

From Corollary 3, it follows immediately that the restriction of a treedepth decomposition to a set is unique up to isomorphism. Importantly, the number of vertices in such a forest is at most $|X| \cdot t$, where $t$ is the treedepth of the graph. This follows since every leaf of the forest is an element of $X$ and the number of vertices from any root to leaf path is at most $t$.

Lemma 7. Let $(F, X, h)$ be a partial decomposition and let $\left(F^{\prime}, X^{\prime}, h^{\prime}\right)$ be the restriction of $(F, X, h)$ to $X^{\prime} \subseteq X$. Then for all $v \in F^{\prime}$ it holds that $h^{\prime}(v)=h(v)$. Furthermore, height $\left(F^{\prime}, X^{\prime}, h^{\prime}\right)=h e i g h t(F, X, h)$.

Proof. By the definition of a restriction: the height function of $\left(F^{\prime}, X^{\prime}, h^{\prime}\right)$ is defined as the restriction of $h$ to $V\left(F^{\prime}\right)$. As such, for all $v \in V\left(F^{\prime}\right)$ we 
have that $h^{\prime}(v)=h(v)$. Also, height $\left(F^{\prime}, X^{\prime}, h^{\prime}\right)=\max _{x \in \operatorname{root}\left(F^{\prime}\right)} h^{\prime}(x)$, and since the sets of root nodes of the forests $F$ and $F^{\prime}$ are the same, we have: $\operatorname{height}\left(F^{\prime}, X^{\prime}, h^{\prime}\right)=\operatorname{height}(F, X, h)$.

Corollary 4. Let $(F, X, h)$ be a restriction of a treedepth decomposition $T$ of the graph $G$ such that $X \neq \emptyset$. Then the the height of $(F, X, h)$ is equal to the height of $T$.

Proof. Follows immediately from Definition 17 and Lemma 7.

Lemma 8. Let $T$ be a (not trivially improvable) treedepth decomposition of a graph $G, X^{\prime} \subseteq X \subseteq V(G)$, and, let $F$ and $F^{\prime}$ be the forests of the decomposition $T$ when restricted to the sets $X$ and $X^{\prime}$, respectively. Then $F$ topologically generalizes $F^{\prime}$ under $X^{\prime}$.

Proof. Note that $V\left(F^{\prime}\right) \subseteq V(F)$ and hence the function $f: V\left(F^{\prime}\right) \rightarrow V(F)$ defined by $f(x)=x$ for all $x \in V\left(F^{\prime}\right)$ witnesses that $F$ topologically generalizes $F^{\prime}$.

As seen Algorithm 1, we will use the contents of the bags of a tree decomposition as the set on which we make restrictions on. If we have a restriction of a tree on the set $X$, we would like to be able to bound the size of the restriction by $|X|$. Since we never delete root nodes we cannot directly show that this is the case. We will later see that by working on rooted we can overcome this problem.

Lemma 9. Let $G=(V, E, r)$ be a rooted graph with root $r$. Then there is an optimal treedepth decomposition $T$ of $G$ such that $\operatorname{root}(T)=r$.

Proof. Suppose that $T^{\prime}$ is an optimal treedepth decomposition of $G$ with root $r^{\prime} \neq r$ (since $G$ is connected, $T^{\prime}$ is actually a tree). We assume that $T^{\prime}$ is not trivially improvable so that every node of $T^{\prime}$ is a vertex of $G$. Let $x_{0}, x_{1}, \ldots, x_{p}$ denote the vertices on the $\left(r^{\prime}, r\right)$-path in $T^{\prime}$, where $x_{0}=r^{\prime}$ and $x_{p}=r$. Then note that since $T^{\prime}$ is a treedepth decomposition and $r$ is a universal vertex, for $0 \leqslant i \leqslant p-1, x_{i}$ has exactly one child $x_{i+1}$ in $T^{\prime}$. That is, $T^{\prime}$ consists of the path $r^{\prime}, x_{1}, \ldots, x_{p-1}, r$ with subtrees attached to $r$. Transform $T^{\prime}$ to obtain $T$ by renaming the vertices of the $\left(r^{\prime}, r\right)$-path as follows: for $0 \leqslant i \leqslant p$, map $x_{i}$ to $x_{(i+1)} \bmod (p+1)$. In this transformation, vertex $r$ is made the ancestor of the vertices $r^{\prime}=x_{1}, x_{2}, \ldots, x_{p}$ but all other ancestor-descendant relationships are preserved and hence $T$ is a treedepth decomposition of $G$. Moreover, height $\left(T^{\prime}\right)=\operatorname{height}(T)$, which is what we wished to prove. 
Lemma 10. Let $G$ be a rooted graph obtained by adding a universal vertex $r$ to a graph $G^{\prime}$. Then $\operatorname{td}(G)=\operatorname{td}\left(G^{\prime}\right)+1$.

Proof. To see that $\mathbf{t d}(G) \leqslant \mathbf{t d}\left(G^{\prime}\right)+1$, take any optimal treedepth decomposition $T^{\prime}$ of $G^{\prime}$ and add edges between $r$ and the roots of the forest of $T^{\prime}$. This yields a treedepth decomposition of $G$ of height $\operatorname{td}\left(G^{\prime}\right)+1$. To see that $\operatorname{td}\left(G^{\prime}\right) \leqslant \operatorname{td}(G)-1$, take an optimal treedepth decomposition $T$ of $G$ with $\operatorname{root}(T)=r$ (Lemma 9] guarantees the existence of such a decomposition). Now delete $r$ from $T$ to obtain a treedepth decomposition of $G^{\prime}$.

Lemma 9 motivates the following definition of treedepth decompositions of rooted graphs.

Definition 18 (Treedepth Decomposition of a Rooted Graph). A treedepth decomposition $T$ of a rooted graph $G=(V, E, r)$ is a treedepth decomposition of $G$ such that $\operatorname{root}(T)=r$.

Every entry in our table during the dynamic programming algorithm will be a restriction of a treedepth decomposition. Let us define some operations on partial decompositions, which we will later use to build our tables during the dynamic programming.

Lemma 11. Let $G=(V, E, r)$ be a rooted graph, let $G^{\prime}=\left(V^{\prime}, E^{\prime}, r\right)$ be a rooted subgraph of $G$ and $X \subseteq V\left(G^{\prime}\right)$ be a set of nodes. Further, let $T$ be nice treedepth decomposition of $G$, and $T^{\prime}$ be a nice treedepth decomposition of $G^{\prime}$ computed from $T$ as in Corollary 2. Let $(F, X, h),\left(F^{\prime}, X, h^{\prime}\right)$ be respective restrictions of $T, T^{\prime}$ to $X$. Then for every pair of functions $\psi$ and $\psi^{\prime}$ that witness that $(F, X, h)$ is a restriction of $T$ to $X$ and $\left(F^{\prime}, X, h^{\prime}\right)$ is a restriction of $T^{\prime}$ to $X$ respectively, it holds that $\psi^{\prime}\left(F^{\prime}\right) \subseteq \psi(F)$.

Proof. Assume to the contrary that there exist functions $\psi, \psi^{\prime}$ such that there exists $v \in F^{\prime}$ with $\psi^{\prime}(v) \notin \psi(F)$. First note that $v \notin X$ as $X \subseteq \psi(F)$. Since $v$ is retained in $F^{\prime}$, there exists a successor $y \in X$ of $v$ in $F^{\prime}$. But by Corollary 2, the ancestor relationship of vertices in $T^{\prime}$ is preserved in $T$, therefore $y$ is also a successor of $v$ in $T$. But then, by construction of $F$, the vertex $v$ must also be contained in $\psi(F)$.

We are now going to prove certain properties of the algorithm, that together will lead us to our main theorem. 
Lemma 12. Let Algorithm 圆 be called on $(G, t, \mathcal{T}, X)$, where $G$ is a graph rooted at $r$, the remaining parameters $t, \mathcal{T}, X$ are as described in the algorithm. Then for every nice treedepth decomposition $T$ of height at most $t$ (that is rooted at $r$ ) of $G\left[V\left(\mathcal{T}_{X}\right)\right]$, the set $R$ returned by the algorithm contains a restriction of $T$ to the set $X$.

Proof. We will prove this by structural induction over tree decompositions: Consider the case that the tree decompositions consists of a single leaf bag. Remember that Algorithm 2 works on nice tree decompositions whose leafs contain a single vertex. The returned set $R$ then consists of the unique partial decomposition for a graph with a single vertex.

Forget case If $X$ is a forget bag whose single child in $\mathcal{T}$ is the bag $X^{\prime}$, then the if-clause at line 7 is entered. By induction hypothesis, we assume that $R^{\prime}$ contains a restriction to the set $X^{\prime}$ of every nice treedepth decomposition $T$ rooted at $r$ of the graph $G\left[V\left(\mathcal{T}_{X^{\prime}}\right)\right]$. Fix such a $T$ and let $\left(F^{\prime}, X^{\prime}, h^{\prime}\right) \in R^{\prime}$ be a restriction of $T$ to the set $X^{\prime}$. Notice that $G\left[V\left(\mathcal{T}_{X^{\prime}}\right)\right]=G\left[V\left(\mathcal{T}_{X}\right)\right]$. Therefore by Corollary 3 , we can restrict $\left(F^{\prime}, X^{\prime}, h^{\prime}\right)$ to the set $X$ to obtain the restriction $(F, X, h)$ of $T$ to $X$. By the definition of the forget operation (Definition 14) the restriction of $\left(F^{\prime}, X^{\prime}, h^{\prime}\right)$ to $X$ is added to $R$.

Introduce case If $X$ is an introduce bag whose single child in $\mathcal{T}$ is the bag $X^{\prime}$, then the if-clause at line 12 is entered. Fix a nice treedepth decomposition $T$ rooted at $r$ of the graph $G\left[V\left(\mathcal{T}_{X}\right)\right]$ of height at most $t$. We want to show that a tuple $(F, X, h)$ is contained in $R$, which is a restriction of $T$ to $X$. Note that the treedepth decomposition $T$ is also a treedepth decomposition of $G\left[V\left(\mathcal{T}_{X^{\prime}}\right)\right]$. Let $T^{\prime}$ be the nice treedepth decomposition of $G\left[V\left(\mathcal{T}_{X^{\prime}}\right)\right]$ computed from $T$ using Corollary 2, By induction hypothesis we assume that $R^{\prime}$ contains the restriction $\left(F^{\prime}, X^{\prime}, h^{\prime}\right)$ of $T^{\prime}$ to $X^{\prime}$. Since $(F, X, h)$ is a restriction, the height of $F$ is at most $t$, its leaves are in $X$ and $r$ is its only root. Therefore $F$ has at most $|X| \cdot t$ vertices. This means that at some point the introduce operation will generate $F$, since all trees which comply with these characteristics are enumerated. By Lemma 8 the tree $F$ topologically generalizes $F^{\prime}$. Thus a tuple $(F, X, h)$ will be added to the set $R$ of the introduce function from Definition [15, and it is left to show that $h$ is computed correctly.

First let us prove that $u$ (the introduced vertex) either is an internal node of $F$ or its parent $y$ is contained in $X$. Let us assume that $u$ is not an 
internal node of $F$, i.e. it is a leaf of $F$, and that $y$ is not an element in $X$. By the properties of tree decompositions, the vertex $u$ can only have edges to vertices in $X$ in the graph $G\left[V\left(\mathcal{T}_{X}\right)\right]$. This means that $u$ must be a leaf in $T$ : If $u$ had a subtree, it would not contain a vertex which is connected to $u$ in $G\left[V\left(\mathcal{T}_{X}\right)\right]$. This would by Lemma 4 contradict our assumption that $T$ is a nice treedepth decomposition. But if we assume that the parent $y$ of $u$ is not an element of $X$, the subtree $T_{y}$ of $T$ rooted at $y$ would induce more than one connected component in $G\left[V\left(\mathcal{T}_{X}\right)\right]$, since $u$ cannot be connected to any node of $V\left(T_{y}\right) \backslash\{y\}$. Thus $u$ has the stated property.

We will now show that the height function $h$ is computed correctly for the leaves of $F$. Let $z$ be a leaf of $F$. As stated before if $z=u$ then $z$ must be a leaf in $T$. For this case we correctly set the height of $z=u$ to one, since it has no children in $F$ either. For $z \neq u$ we know that the subtree $T_{z}$ of $T$ rooted at $z$ forms a single connected component in $G\left[V\left(\mathcal{T}_{X}\right)\right]$. Since $u \notin V\left(T_{z}\right)$ it follows that $T_{z}$ is still a single connected component in $G\left[V\left(\mathcal{T}_{X^{\prime}}\right)\right]$. Thus $T_{z}$ is also a subtree of $T^{\prime}$. Accordingly we set the value correctly to $h(z)=$ height $_{T^{\prime}}(z)=h^{\prime}(z)$.

We have shown that we set the correct height for the leaves of $F$, let us now show inductively that the height is also set correctly for any internal node $z$ of $F$. By induction we can assume that the height is set correctly for all children of $z$ in $F$. Let $C$ be the set of children of $z$ in $T$ which are not elements of $F$. By Lemma 5, if we construct a tree $T_{z}^{C}$ from all the subtrees rooted at a node in $C$ with $z$ added as its root, the graph $G\left[V\left(T_{z}^{C}\right)\right]$ is connected. Since $u$ cannot be an element of this tree, it follows that $G\left[V\left(T_{z}^{C}\right)\right]$ is a connected subgraph of $G\left[V\left(\mathcal{T}_{X^{\prime}}\right)\right]$. From the computation of $T^{\prime}$ defined in Corollary 2 it follows that $T_{z}^{C}$ is a subtree of $T^{\prime}$. Therefore height $T_{T}(x)=$ height $_{T^{\prime}}(x)$ for $x \in C$. The height of $z$ is height $(c)+1$ where $c$ is a child of $z$ in $T$. Consider the case where $c \in C$ : By Corollary 2 we know that height of any such $c$ cannot be greater in $T^{\prime}$ than in $T$, thus if height ${ }_{T}(z)=$ height $_{T}(c)+1$ it follows that height ${ }_{T^{\prime}}(z)=\operatorname{height}_{T^{\prime}}(c)+1=h^{\prime}(z)$. Otherwise, if $c \in C$, by inductive hypothesis $h(c)=$ height $_{T}(c)$ which we use to set the value of $h(z)$.

Join case Finally, if $X$ is a join bag with two children $X_{1}$ and $X_{2}$ in $\mathcal{T}$, then the if-clause at line 17 is entered. Let again $T$ be a nice treedepth decomposition rooted at $r$ of the graph $G\left[V\left(\mathcal{T}_{X}\right)\right]$. Then $T$ is also treedepth decomposition of both $G\left[V\left(\mathcal{T}_{X_{1}}\right)\right]$ and $G\left[V\left(\mathcal{T}_{X_{2}}\right)\right]$. Notice that by the properties of tree decompositions, $V\left(\mathcal{T}_{X_{1}}\right) \cap V\left(\mathcal{T}_{X_{2}}\right)=X$. Let $(F, X, h)$ be the 
restriction of $T$ to the set $X$ for the graph $G\left[V\left(\mathcal{T}_{X}\right)\right]$. Furthermore, let $T_{1}$ and $T_{2}$ be nice treedepth decomposition computed from $T$ by Corollary 2 for the graphs $G\left[V\left(\mathcal{T}_{X_{1}}\right)\right]$ and $G\left[V\left(\mathcal{T}_{X_{2}}\right)\right]$ respectively. Furthermore let $\left(F_{1}, X, h_{1}\right)$ be the restriction of $T_{1}$ to $X$ and $\left(F_{2}, X, h_{2}\right)$ the restriction of $T_{2}$ to $X$. By inductive hypothesis, $\left(F_{1}, X, h_{1}\right) \in R_{1}$ and $\left(F_{2}, X, h_{2}\right) \in R_{2}$. Note that this is because $X=X_{1}=X_{2}$. At some point the introduce operation will generate $F$ for the same reason as in the introduce case. By Lemma 8 is both a topological generalization of $F_{1}$ and $F_{2}$. We now need to show that there exist two witness functions $f_{1}$ and $f_{2}$ respectively such that the intersection of their images is exactly $X$.

Let $\psi, \psi_{1}, \psi_{2}$ witness that $(F, X, h),\left(F_{1}, X, X_{1}\right),\left(F_{2}, X, h_{2}\right)$ are restrictions of $T, T_{1}, T_{2}$ to $X$, respectively. By Lemma 11 we have that $\psi_{1}\left(F_{1}\right) \subseteq$ $\psi(F)$ and $\psi_{2}\left(F_{2}\right) \subseteq \psi(F)$. Therefore we can construct $f_{1}=\psi^{-1} \circ \psi_{1}$ and $f_{2}=\psi^{-1} \circ \psi_{2}$, both of which are well-defined as $\psi$ is injective. It remains to show that $f_{1}\left(F_{1}\right) \cap f_{2}\left(F_{2}\right)=X$. By construction we already see that $f_{1}(X)=f_{2}(X)=X$. Since $\psi_{1}\left(F_{1}\right) \subseteq T_{1}$ and $\psi_{2}\left(F_{2}\right) \subseteq T_{2}$ with $V\left(T_{1}\right) \cap V\left(T_{2}\right)=X$, the claim follows. Since $f_{1}, f_{2}$ exist, the join operation will generate them at some point. Therefore a partial decomposition whose tree is $F$ will be added to the result set. It remains to show that the height function as computed in the join operation is correct.

Let us first show the following: let $z \in V(T) \backslash V(F)$ be a node whose parent is contained in $F$. Then either $V\left(T_{z}\right) \cap V\left(\mathcal{T}_{X_{1}}\right)=\emptyset$ or $V\left(T_{z}\right) \cap V\left(\mathcal{T}_{X_{2}}\right)=$ $\emptyset$. Assume to the contrary that $T_{z}$ contains vertices of both $V\left(\mathcal{T}_{X_{1}}\right)$ and $V\left(\mathcal{T}_{X_{2}}\right)$. Since $X$ separates these two sets in $G\left[V\left(\mathcal{T}_{X}\right)\right]$ and by assumption no vertex of $X$ is contained in $T_{z}$, this implies that $G\left[V\left(T_{z}\right)\right]$ has more than one connected component. But this contradicts $T$ being a nice treedepth decomposition.

The remaining proof parallels the proof for the introduce case. Let $z$ be a leaf of $F, C_{1}$ be the set of children contained in $V\left(\mathcal{T}_{X_{1}}\right)$ and $C_{2}$ the set of children contained in $V\left(\mathcal{T}_{X_{2}}\right)$. Notice that since $z$ is a leaf of $F$, the set $C_{1} \cup C_{2}$ does not contain any element of $X$. By Lemma [5, the tree $T_{z}^{C_{1}}$ induces a connected subgraph in $G\left[V\left(\mathcal{T}_{X_{1}}\right)\right]$ and the tree $T_{z}^{C_{2}}$ induces a connected subgraph in $G\left[V\left(\mathcal{T}_{X_{2}}\right)\right]$. As proved earlier in the introduce case, the trees $T_{z}^{C_{1}}, T_{z}^{C_{2}}$ are subtrees of $T_{1}$ and $T_{2}$, respectively.

We will now show that the height function $h$ is computed correctly for the leaves of $F$. Let $z$ be a leaf of $F$. The height of $z$ in $T$ is either the height of $T_{z}^{C_{1}}$ or of $T_{z}^{C_{2}}$. By the previous observation these two trees are subtrees of respectively $T_{1}$ and $T_{2}$, therefore their heights are given by height $T_{T_{1}}(z)$ and 
height $_{T_{2}}(z)$. As the height of $z$ is computed as $h(z)=\max \left\{h_{1}(z), h_{2}(z)\right\}$ which is exactly $\max \left\{\right.$ height $_{T_{1}}(z)$, height $\left._{T_{2}}(z)\right\}$, we conclude that the height of the leaves of $F$ is correct.

We can now prove inductively that the height $h(z)$ for any internal node $z$ is also computed correctly. Let $C$ be the set of children of $z$ in $T$ which are not nodes of $F$. Define $C_{1}=C \cap V\left(\mathcal{T}_{X_{1}}\right)$ and $C_{2}=C \cap V\left(\mathcal{T}_{X_{2}}\right)$, both of which could potentially be empty. As previously stated, $T_{z}^{C_{1}}$ and $T_{z}^{C_{2}}$ induce connected subgraphs in $G\left[V\left(\mathcal{T}_{X_{1}}\right)\right]$ and $G\left[V\left(\mathcal{T}_{X_{2}}\right)\right]$ and are subtrees of $T_{1}$ and $T_{2}$, respectively. From Corollary 2 we know that the height of $z$ in $T$ is at least the height of $z$ in $T_{1}$ (if it is contained in $T_{1}$ ) and the height of $z$ in $T_{2}$ (if it is contained in $T_{2}$ ).

Thus it follows that if height $T_{T}(z)=$ height $_{T_{z}^{C_{1}}}(z)$, then height $T_{T_{1}}(z)=$ height $_{T_{z}^{C_{1}}}(z)$. Analogously, if $\operatorname{height}_{T}(z)=\operatorname{height}_{T_{z}^{C_{2}}}(z)$, then height ${ }_{T_{2}}(z)=$ height $_{T_{z} C_{2}}(z)$. Taking the maximum $h_{1}\left(f_{1}^{-1}(z)\right)$ and $h_{2}\left(f_{2}^{-1}(z)\right)$ (if the inverse values exist) and all the children of $z$ in $F$ therefore yields the correct value for $h(z)$.

Since these are all the possible execution paths of the algorithm, it follows by induction that the lemma is correct.

We have now shown that our algorithm will contain a partial decomposition representing any nice treedepth decomposition of height at most $t$. This is not sufficient to proof the correctness of the algorithm since our tables could still contain partial decomposition which are not restrictions of treedepth decompositions of height at most $t$. The next lemma proofs precisely that this is not the case.

Lemma 13. Let Algorithm 2 be called on $(G, t, \mathcal{T}, X)$, where $G$ is a graph rooted at $r$, the remaining parameters $t, \mathcal{T}, X$ are as described in the algorithm. Then every member of $R$ returned by the algorithm is a restriction of a treedepth decompositions of $G\left[V\left(\mathcal{T}_{X}\right)\right]$ to $X$.

Proof. We will prove this by structural induction over tree decompositions: Consider the case that the tree decomposition consists of a single leaf bag containing only a single vertex. The returned set $R$ then consists of the unique partial decomposition for this graph.

Forget case For the forget case, the correctness of the statement follows directly from Lemma 6 using the induction hypothesis. 
Introduce case Consider the case that the bag $X$ with single child $X^{\prime}$ introduces the vertex $u$. The set $R^{\prime}$ contains, by induction hypothesis, only restrictions of treedepth decompositions. We have to show that the operation of introducing $u$ generates only restrictions of treedepth decompositions. Consider any $(F, X, h) \in R$. First let us show that every edge incident to $u$ in $G\left[V\left(\mathcal{T}_{X}\right)\right]$ is contained in $\operatorname{clos}(F)$. Because $X^{\prime}$ separates $u$ from $G\left[V\left(\mathcal{T}_{X^{\prime}}\right) \backslash X\right]$, any such edge has its other endpoint necessarily in $X^{\prime}$. Since the introduce operation by construction only returns restrictions with $E(G[X]) \subseteq E(\operatorname{clos}(F)[X])$, we conclude that every edge incident to $u$ in $G\left[V\left(\mathcal{T}_{X}\right)\right]$ is contained in the closure of $F$.

Consider $\left(F^{\prime}, X^{\prime}, h^{\prime}\right) \in R^{\prime}$ such that $F$ topologically generalizes $F^{\prime}$ and further such that $(F, X, h) \in$ intro $_{t}\left(\left\{\left(F^{\prime}, X^{\prime}, h^{\prime}\right)\right\}, X^{\prime}, u, G\right)$. Such a restriction must, by the definition of the introduce operation, exist and by induction hypothesis is a restriction of a treedepth decomposition $T^{\prime}$ of $G\left[V\left(\mathcal{T}_{X^{\prime}}\right)\right]$. Note that every edge $v w \in E\left(G\left[V\left(\mathcal{T}_{X}\right)\right]\right)$ with $v \neq u \neq w$ is by induction hypothesis contained in the closure of $T^{\prime}$.

We will now show that we can construct a treedepth decomposition $T$ of $G\left[V\left(\mathcal{T}_{X}\right)\right]$ from $T^{\prime}$ of which $(F, X, h)$ is a restriction. Let $\psi^{\prime}$ witness that $\left(F^{\prime}, X^{\prime}, h^{\prime}\right)$ is a restriction of $T^{\prime}$ to $X^{\prime}$. Let $f: V\left(F^{\prime}\right) \rightarrow V(F)$ be a function that witnesses that $F$ topologically generalizes $F^{\prime}$ with $u \notin f\left(F^{\prime}\right)$. We first construct $(\hat{F}, X, \hat{h}),\left(\hat{F}^{\prime}, X^{\prime}, \hat{h}^{\prime}\right)$ which are equivalent to $(F, X, h),\left(F^{\prime}, X^{\prime}, h^{\prime}\right)$, respectively, such that $V\left(\hat{F}^{\prime}\right) \subset V(\hat{F}) \subseteq V\left(T^{\prime}\right) \cup\{u\}$ and so that the function $f$ carried over to $\hat{F}^{\prime}, \hat{F}$ is simply the identity.

By Definition [17, there exists $\hat{F}^{\prime} \subseteq T^{\prime}$ and $\hat{h}^{\prime}$ such that $\left(\hat{F}^{\prime}, X^{\prime}, \hat{h}^{\prime}\right)$ is a restriction of $T^{\prime}$ to $X^{\prime}$. Let $\hat{\psi}^{\prime}: V\left(\hat{F}^{\prime}\right) \rightarrow V\left(F^{\prime}\right)$ be the function that witnesses the equivalency of $\left(\hat{F}^{\prime}, X^{\prime}, \hat{h}^{\prime}\right)$ and $\left(F^{\prime}, X^{\prime}, h^{\prime}\right)$. Then $\hat{F}$ is the tree with nodes $V(\hat{F})=V\left(\hat{F}^{\prime}\right) \cup\{u\}$ isomorphic to $F$ where the isomorphism is witnessed by the bijection $\phi: \hat{F} \rightarrow F$ defined via

$$
\phi(v)= \begin{cases}v & \text { for } v=u \\ f\left(\hat{\psi}^{\prime}(v)\right) & \text { otherwise }\end{cases}
$$

and $\hat{h}=h \circ \phi$. We finally construct $T$ as follows: take the rooted forest $T^{\prime} \backslash \hat{F}^{\prime}$ and add $\hat{F}$ to it, then add the edge set $\left\{x y \in E\left(T^{\prime}\right) \mid x \in \hat{F}^{\prime}, y \notin \hat{F}^{\prime}\right\}$.

Let us first verify that $(\hat{F}, X, \hat{h})$, and thus by equivalency also $(F, X, h)$, is indeed a restriction of $T$ to $X$. By construction it is immediately apparent that the iterative deletion of leaves of $T$ not in $X$ indeed yields the tree $\hat{F}$. 
However, we also need to verify that the height function $\hat{h}$ is correct, i.e. that for all $v \in \hat{F}, \hat{h}(v)=\operatorname{height}_{T}(v)$.

We prove the correctness of $\hat{h}$ inductively beginning at the leaves of $\hat{F}$ : consider a leaf $v \in \hat{F}$ with $v \neq u$. The introduce operation calculates $h$ as $h(\phi(v))=h^{\prime}\left(\hat{\psi}^{\prime}(v)\right)$ and thus $\hat{h}$ as $\hat{h}(v)=\hat{h}^{\prime}(v)$. By construction, $v$ in $T$ inherits the subtrees of $v$ in $T^{\prime}$, thus height $T_{T}(v)=\operatorname{height}_{T^{\prime}}(v)=\hat{h}(v)$. Next assume $u$ is a leaf in $\hat{F}$ : then the introduce operation sets $\hat{h}(v)=1$. By construction of $T, u$ will then not have any children and we conclude that $\operatorname{height}_{T}(u)=\hat{h}(u)$ in this case. The statement now follows by induction: consider any internal node $v \in \hat{F}, v \neq u$ with children $C$ in $T$. Let $C^{\prime}$ be the set of children of $v$ in $T^{\prime}$. By induction hypothesis, for all $w \in C \cap V(\hat{F})$, $\operatorname{height}_{T}(w)=\hat{h}(w)$. By construction of $T$ and the fact that $\hat{F}$ is a topological generalization of $\hat{F}^{\prime}$, it holds that

$$
\begin{aligned}
& \max _{w \in C \backslash V(\hat{F})} \operatorname{height}_{T}(w)=\max _{w \in C^{\prime} \backslash V\left(\hat{F}^{\prime}\right)} \text { height }_{T^{\prime}}(w) \\
& \max _{w \in C^{\prime} \cap V\left(\hat{F}^{\prime}\right)} \operatorname{height}_{T^{\prime}}(w) \leqslant \max _{w \in C \cap V(\hat{F})} \operatorname{height}_{T}(w)
\end{aligned}
$$

Further note that

$$
\begin{aligned}
\hat{h}^{\prime}(v)-1 & =\max _{w \in C^{\prime}} \operatorname{height}_{T^{\prime}}(w) \\
& =\max \left\{\max _{w \in C^{\prime} \backslash V\left(\hat{F}^{\prime}\right)} \operatorname{height}_{T^{\prime}}(w), \max _{w \in C^{\prime} \cap V\left(\hat{F}^{\prime}\right)} \operatorname{height}_{T^{\prime}}(w)\right\}
\end{aligned}
$$

Therefore it holds that

$$
\begin{aligned}
\max _{w \in C} \operatorname{height}_{T}(w) & =\max \left\{\max _{w \in C \backslash V(\hat{F})} \operatorname{height}_{T}(w), \max _{w \in C \cap V(\hat{F})} \operatorname{height}_{T}(w)\right\} & \\
& =\max \left\{\max _{w \in C^{\prime} \backslash V\left(\hat{F}^{\prime}\right)} \operatorname{height}_{T^{\prime}}(w), \max _{w \in C \cap V(\hat{F})} \operatorname{height}_{T}(w)\right\} & \text { by (1) } \\
= & \max \left\{\max _{w \in C^{\prime} \backslash V\left(\hat{F}^{\prime}\right)} \operatorname{height}_{T^{\prime}}(w), \max _{w \in C^{\prime} \cap V\left(\hat{F}^{\prime}\right)} \operatorname{height}_{T^{\prime}}(w),\right. & \\
& \left.\max _{w \in C \cap V(\hat{F})} \operatorname{height}_{T}(w)\right\} & \text { by (2) } \\
= & \max \left\{\hat{h}^{\prime}(v)-1, \max _{w \in C \cap V(\hat{F})} \operatorname{height}_{T}(w)\right\} & \\
= & \max \left\{\hat{h}^{\prime}(v)-1, \max _{w \in C \cap V(\hat{F})} \hat{h}(w)\right\} & \\
= & \hat{h}(v)-1 & \text { by introduce operation }
\end{aligned}
$$


The proof for $\hat{h}(u)$ works analogously, with the slight difference that $u$ will not have any children that are not in $\hat{F}$. We conclude that $(\hat{F}, X, \hat{h})$ and therefore $(F, X, h)$ is a restriction of $T$ to $X$.

It remains to show that $T$ is a treedepth decomposition of $G\left[V\left(\mathcal{T}_{X}\right)\right]$. Note that $V(T)=V\left(\mathcal{T}_{X}\right)$. By construction of $F$ and thus $\hat{F}$, edges incident to $u$ are contained in $\operatorname{clos}(\hat{F})$ and thus in $\cos (T)$. Since $\hat{F}$ is a topological generalization of $\hat{F}^{\prime}, \operatorname{clos}\left(\hat{F}^{\prime}\right) \subseteq \operatorname{clos}(\hat{F})$ and therefore every edge of $G\left[V\left(\mathcal{T}_{X}\right)\right]$ that lives in $V\left(\hat{F}^{\prime}\right)$ is contained in the closure of $T$. As $T^{\prime} \backslash \hat{F}^{\prime}$ is a subgraph of $T$, the edges contained in $\operatorname{clos}\left(T^{\prime} \backslash \hat{F}^{\prime}\right)$ are contained in $\operatorname{clos}(T)$. It remains to show that every edge $x y$ that has one endpoint $x \in \hat{F}^{\prime}$ and the other endpoint $y \in T^{\prime} \backslash \hat{F}^{\prime}$ will also be covered by the closure of $T$. Consider the $x$-y-path in $T^{\prime}$ : this path contains a node $z \in \hat{F}^{\prime}$ whose successor is not contained in $\hat{F}^{\prime}$. Because $\hat{F}$ is a topological generalization of $\hat{F}^{\prime}$, the node $x$ is an ancestor of $z$ in $\hat{F}$ and thus in $T$. Furthermore, by construction of $T$, the node $z$ is an ancestor of $y$ in $T$; it follows by transitivity that $x y \in \cos (T)$. Therefore $T$ is a treedepth decomposition of $G\left[V\left(\mathcal{T}_{X}\right)\right]$ and the lemma follows for the introduce-case.

Join case Consider the case of a bag $X$ with children $X_{1}=X_{2}=X$. The sets $R_{1}, R_{2}$ contain, by induction hypothesis, only restrictions of treedepth decompositions. We have to show that the operation of joining $X_{1}, X_{2}$ generates only restrictions of treedepth decompositions. Consider any $(F, X, h) \in$ $R,\left(F_{1}, X, h_{1}\right) \in R_{1},\left(F_{2}, X, h_{2}\right) \in R_{2}$ such that $F$ topologically generalizes $F_{1}$ and $F_{2}$ and further $(F, X, h) \in$ join $_{t}\left(X, R_{1}, R_{2}, G\right)$. such restrictions must, by the definition of the join operation, exist and by induction hypothesis they are restrictions of treedepth decompositions $T_{1}, T_{2}$ of $G\left[V\left(\mathcal{T}_{X_{1}}\right)\right], G\left[V\left(\mathcal{T}_{X_{2}}\right)\right]$, respectively. Note that every edge $v w \in E\left(G\left[V\left(\mathcal{T}_{X}\right)\right]\right)$ is by induction hypothesis contained either in the closure of $T_{1}$ or the closure of $T_{2}$.

We will now show that we can construct a treedepth decomposition $T$ of $G\left[V\left(\mathcal{T}_{X}\right)\right]$ from $T_{1}, T_{2}$ of which $(F, X, h)$ is a restriction.

For $i \in\{1,2\}$, let $\psi_{i}$ witness that $\left(F_{i}, X, h_{i}\right)$ is a restriction of $T_{i}$ to $X_{i}$. Let $f_{i}: V\left(F_{i}\right) \rightarrow V(F)$ be a function that witnesses that $F$ topologically generalizes $F_{i}$. We first construct $(\hat{F}, X, \hat{h}),\left(\hat{F}_{i}, X, \hat{h}_{i}\right), i \in\{1,2\}$ which are equivalent to $(F, X, h),\left(F_{i}, X, h_{i}\right)$, respectively, such that $V\left(\hat{F}_{i}\right) \subseteq V(\hat{F}) \subseteq$ $V\left(T_{1}\right) \cup V\left(T_{2}\right)$ and so that the functions $f_{i}$ that witness the topological generalization of $F_{i}$, by $F$ simply become the identity on $\hat{F}_{i}, \hat{F}$.

By Definition [17, there exists $\hat{F}_{i} \subseteq T_{i}$ and $\hat{h}_{i}$ such that $\left(\hat{F}_{i}, X, \hat{h}_{i}\right)$ is a 
restriction of $T_{i}$ to $X_{i}=X$.

Let $\hat{\psi}_{i}: V\left(\hat{F}_{i}\right) \rightarrow V\left(F_{i}\right)$ be the function that witnesses the equivalency of $\left(\hat{F}_{i}, X, \hat{h}_{i}\right)$ and $\left(F_{i}, X, h_{i}\right)$. Then $\hat{F}$ is the tree with nodes $V(\hat{F})=V\left(\hat{F}_{1}\right) \cup$ $V\left(\hat{F}_{2}\right)$ isomorphic to $F$ where the isomorphism is witnessed by the bijection $\phi: \hat{F} \rightarrow F$ defined via

$$
\phi(v)=f_{i}\left(\hat{\psi}_{i}(v)\right) \quad v \in V\left(\hat{F}_{i}\right)
$$

where we use the fact that for any $v \in X, \hat{\psi}_{i}(v)=v$ and $f_{i}(v)=v$. We further set $\hat{h}=h \circ \phi$. We finally construct $T$ as follows: take the union of the rooted forests $T_{1} \backslash \hat{F}_{1}, T_{2} \backslash \hat{F}_{2}$ and $\hat{F}$, then add for $i \in\{1,2\}$ the edge sets $\left\{x y \in E\left(T_{i}\right) \mid x \in \hat{F}_{i}, y \notin \hat{F}_{i}\right\}$.

Let us first verify that $(\hat{F}, X, \hat{h})$, and thus by equivalency also $(F, X, h)$, is indeed a restriction of $T$ to $X$. By construction it is immediately apparent that the iterative deletion of leaves of $T$ not in $X$ indeed yields the tree $\hat{F}$. However, we also need to verify that the height function $\hat{h}$ is correct, i.e. that for all $v \in \hat{F}, \hat{h}(v)=\operatorname{height}_{T}(v)$.

We prove the correctness of $\hat{h}$ inductively beginning at the leaves of $\hat{F}$ : consider a leaf $v \in \hat{F}$. Since $v \in X$, the join operation calculates $h$ as $h(v)=\max _{i \in\{1,2\}} h_{i}(v)$ and thus $\hat{h}$ as $\hat{h}(v)=\max _{i \in\{1,2\}} h_{i}(v)$. By construction, $v$ in $T$ inherits the subtrees of $v$ in $T_{1}$ and of $v$ in $T_{2}$, thus $\operatorname{height}_{T}(v)=\max _{i \in\{1,2\}} \operatorname{height}_{T_{i}}(v)=\hat{h}(v)$. The statement now follows by induction: consider any internal node $v \in \hat{F}$ with children $C$ in $T$. For $i \in\{1,2\}$, let $C_{i}$ be the set of children of $v$ in $T_{i}$. By induction hypothesis, for all $w \in C \cap V(\hat{F})$, height ${ }_{T}(w)=\hat{h}(w)$. By construction of $T$ and the fact that $\hat{F}$ is a topological generalization of $\hat{F}_{1}, \hat{F}_{2}$, it holds that

$$
\begin{aligned}
\max _{w \in C \backslash V(\hat{F})} \operatorname{height}_{T}(w) & =\max _{i \in\{1,2\}} \max _{w \in C_{i} \backslash V\left(\hat{F}_{i}\right)} \operatorname{height}_{T_{i}}(w) \\
\max _{i \in\{1,2\}} \max _{w \in C_{i} \cap V\left(\hat{F}_{i}\right)} \operatorname{height}_{T_{i}}(w) & \leqslant \max _{w \in C \cap V(\hat{F})} \operatorname{height}_{T}(w)
\end{aligned}
$$

Further note that

$$
\begin{aligned}
\hat{h}_{i}(v)-1 & =\max _{w \in C_{i}} \operatorname{height}_{T_{i}}(w) \\
& =\max \left\{\max _{w \in C_{i} \backslash V\left(\hat{F}_{i}\right)} \operatorname{height}_{T_{i}}(w), \max _{w \in C_{i} \cap V\left(\hat{F}_{i}\right)} \operatorname{height}_{T_{i}}(w)\right\}
\end{aligned}
$$


Therefore it holds that

$$
\begin{array}{rlr}
\max _{w \in C} \operatorname{height}_{T}(w)=\max \left\{\max _{w \in C \backslash V(\hat{F})} \operatorname{height}_{T}(w), \max _{w \in C \cap V(\hat{F})} \operatorname{height}_{T}(w)\right\} & \\
= & \max \left\{\max _{i \in\{1,2\}} \max _{w \in C_{i} \backslash V\left(\hat{F}_{i}\right)} \operatorname{height}_{T_{i}}(w), \max _{w \in C \cap V(\hat{F})} \operatorname{height}_{T}(w)\right\} & \text { by (4) } \\
= & \max \left\{\max _{i \in\{1,2\}}\left\{\max _{w \in C_{i} \backslash V\left(\hat{F}_{i}\right)} \operatorname{height}_{T_{i}}(w), \max _{w \in C_{i} \cap V\left(\hat{F}_{i}\right)} \operatorname{height}_{T_{i}}(w)\right\}\right. & \\
\left.\max _{w \in C \cap V(\hat{F})} \operatorname{height}_{T}(w)\right\} & \text { by (5) } \\
= & \max \left\{\max _{i \in\{1,2\}} \hat{h}_{i}(v)-1, \max _{w \in C \cap V(\hat{F})} \operatorname{height}_{T}(w)\right\} & \text { by (6) } \\
= & \max \left\{\max _{i \in\{1,2\}} \hat{h}_{i}(v)-1, \max _{w \in C \cap V(\hat{F})} \hat{h}(w)\right\} & \\
=\hat{h}(v)-1 & \text { by join operation }
\end{array}
$$

It remains to show that $T$ is a treedepth decomposition of $G\left[V\left(\mathcal{T}_{X}\right)\right]$. Note that $V(T)=V\left(\mathcal{T}_{X}\right)$. Since $\hat{F}$ is a topological generalization of $\hat{F}_{i}$ for $i \in\{1,2\}$, it holds that $\operatorname{clos}\left(\hat{F}_{i}\right) \subseteq \operatorname{clos}(\hat{F})$ and therefore every edge of $G\left[V\left(\mathcal{T}_{X}\right)\right]$ that lives in $V\left(\hat{F}_{i}\right)$ is contained in the closure of $T$. As $T_{i} \backslash \hat{F}_{i}$ is by construction a subgraph of $T$, the edges contained in each $\operatorname{clos}\left(T_{i} \backslash \hat{F}_{i}\right)$ are contained in $\operatorname{clos}(T)$. It remains to show that for $i \in\{1,2\}$, every edge $x y$ that has one endpoint $x \in \hat{F}_{i}$ and the other endpoint $y \in T_{i} \backslash \hat{F}_{i}$ will also be covered by the closure of $T$. Consider the $x$-y-path in $T_{i}$ : this path contains a node $z \in \hat{F}_{i}$ whose successor is not contained in $\hat{F}_{i}$. Because $\hat{F}$ is a topological generalization of $\hat{F}_{i}$, the node $x$ is an ancestor of $z$ in $\hat{F}$ and thus in $T$. Furthermore, by construction of $T$, the node $z$ is an ancestor of $y$ in $T$; it follows by transitivity that $x y \in \operatorname{clos}(T)$. Therefore $T$ is a treedepth decomposition of $G\left[V\left(\mathcal{T}_{X}\right)\right]$ and the lemma follows for the introduce-case.

Lemma 14. Algorithm 1 decides the treedepth of the input graph $G^{\prime}$.

Proof. First, it is easy to see that $\mathcal{T}$ is a nice tree decomposition of $G$ of width $t+1$. By Lemma 12 and Lemma 13 it follows that the set $R$ contains all restrictions of any nice treedepth decomposition rooted at $r$ of the rooted graph $G$ after line 6 of Algorithm 2 is executed. From Corollary 4 we know that the height of the partial decomposition equals the height of the treedepth decomposition of which it is a restriction. From Lemma 13 we know that every partial decomposition in $R$ is a restriction of a treedepth decomposition of $G$. From Lemma 3 and Lemma 9 we know that there is a nice treedepth 
decomposition rooted at $r$ of minimal height of the rooted graph $G$. From Lemma 10 we know that $G$ has a treedepth decomposition of height $t+1$ if and only if $G^{\prime}$ has one of height $t$. Thus the return statement at line 7 will give the correct answer.

\subsection{Running time of Algorithm 1}

Lemma 15. For a set $X$, the number of possible restrictions on $X$ of height at most $t$ is, up to equivalency, bounded by $2^{|X| t+|X| \log t+|X| \log |X|}$.

Proof. For any restriction $(F, X, h)$ of height at most $t$, we have that $|F| \leqslant$ $|X| \cdot t$, since every leaf of $F$ is contained in $X$ and height $(F) \leqslant t$.

First note that any monotone path $P$ (i.e. a path on which every node is either an ancestor or a descendant of any other node on the path) inside the forest of a restriction can be labeled by $h$ in at most $2^{t}$ ways: since $h$ will increases strictly while following $P$ from top to bottom and the $|P| \leqslant t$, the function $\left.h\right|_{P}$ is already completely determined by the set $h(P)$.

Consider any ordering $x_{1}, \ldots, x_{|X|}$ of the elements in $X$ and denote by $X_{i}=\left\{x_{1}, \ldots, x_{i}\right\}$, for $1 \leqslant i \leqslant|X|$. We upper-bound the number of restrictions by considering the following construction: given a restriction $\left(F, X_{i}, h_{i}\right)$, we have at most $i \cdot t \cdot 2^{t}$ ways of constructing a restriction on $X_{i+1}$ : we choose one of $i \cdot t$ nodes of $F$ and attach one of the possible $2^{t}$ labeled paths to it, with leaf-node $x_{i+1}$. We allow adding a path of length zero, this operation simply exchanges the initially chosen node with $x_{i+1}$. Clearly all restrictions on $X_{i+1}$ can be generated in such a way from restrictions on $X_{i}$. Thus the number of restrictions is given by

$$
\prod_{i=1}^{|X|} t i 2^{t}=2^{|X| t+|X| \log t}|X| ! \leqslant 2^{|X| t+|X| \log t+|X| \log |X|} .
$$

Lemma 16. Given restrictions $(F, X, h),\left(F^{\prime}, X^{\prime}, h^{\prime}\right)$ with $X^{\prime} \subseteq X$ there are at most $2^{t \cdot\left|X^{\prime}\right| / 2}$ ways of how $F$ can topologically generalize $F^{\prime}$ and in this time, all candidate maps witnessing this fact can be generated.

Proof. We upper bound the number of possible maps $f$ that witness that $F$ is a topological generalization of $F^{\prime}$. Consider a leaf node $v \in F^{\prime}$, which

necessarily is contained in $v \in X^{\prime} \subseteq X$. Let $P_{v}^{\prime}$ be the path from the root 
of $F^{\prime}$ to $v$ (in $F^{\prime}$ ) and $P_{v}$ the path from the root of $F$ to $v$ (in $F$ ). In order for $f$ to preserve the ancestor relationship of vertices in $F^{\prime}$, the vertices of $P_{v}^{\prime}$ must be mapped to vertices of $P_{v}$ while preserving order, i.e. if $x$ appears before $y$ in $P_{v}^{\prime}$ then $f(x)$ must appear before $f(y)$ in $P_{v}$. It follows that there are exactly $\left(\begin{array}{l}\left|P_{v}\right| \\ \left|P_{v}^{\prime}\right|\end{array}\right)$ ways of how $f$ could map $P_{v}^{\prime}$ to $P_{v}$.

We now upper bound the number of maps by taking the product of all such paths:

$$
\prod_{v \in X^{\prime}}\left(\begin{array}{l}
\left|P_{v}\right| \\
\left|P_{v}^{\prime}\right|
\end{array}\right) \leqslant 2^{t \cdot\left|X^{\prime}\right| / 2}
$$

using the fact that no rooted path in $F$ and $F^{\prime}$ exceeds length $t$. This method can be used constructively (since we can check whether a map indeed witnesses a topological generalization in polynomial time) to enumerate all maps.

Lemma 17. Algorithm 2 called on $G, t, \mathcal{T}$ and $X$, where $G$ is a graph rooted at $r$ of size $n, \mathcal{T}$ is a nice tree decomposition of $G$ of width $w$ where every bag contains $r$ and $X$ is a bag of $\mathcal{T}$ runs in time $O\left(2^{4 w t+3 w \log w t} \cdot w t \cdot n\right)$.

Proof. A nice tree decomposition can only have $O(n)$ bags, therefore the linear dependence follows easily.

By Lemma 15, the set $R$ of restrictions at any given time cannot contain more then $2^{w t+w \log t+w \log w}$ elements. During the join case, we generate all possible restrictions $(F, X, h)$ and for each we consider all pairs $\left(F_{1}, X, h_{1}\right),\left(F_{2}, X, h_{2}\right)$ from the respective tables $R_{1}, R_{2}$ of the child bags. For such a pair we need to compute all possible maps $f_{1}, f_{2}$ that might witness the fact that $F$ topologically generalizes both $F_{1}$ and $F_{2}$. To check if a function witnesses a topological generalization takes linear time in the size of the trees, i.e. $O(t \cdot|X|)$. The total amount of time needed for this operation, using the bound provided by Lemma 16, is at most

$$
\left(2^{w t+w \log t+w \log w}\right)^{3} \cdot\left(2^{t / 2 \cdot w}\right)^{2} \cdot O(w t)=O\left(2^{4 w t+3 w \log w t} \cdot w t\right)
$$

Both forget- and introduce-operation and checking if the result set already contains an equivalent partial decomposition have running times bounded by this function, thus $O\left(2^{4 w t+3 w \log w t} \cdot w t\right)$ is also an upper bound for the total running time of every operation and the lemma follows. 
We finally are able to sum up the results in the following theorem, a direct consequence of Lemma 14 and Lemma 17.

Theorem 1. Let $G$ be a graph of size $n$ and $t$ an integer. Given a tree decomposition of $G$ of width $w$, one can decide in time and space $O\left(2^{4 w t+3 w \log w t}\right.$. $w t \cdot n)$ whether $G$ has treedepth at most $t$ and if so, output a treedepth decomposition of that height.

To actually construct a solution, we keep the tables of all bags in memory and employ backtracking to reconstruct a minimal treedepth decomposition.

\section{A Simple Algorithm}

We can now use Theorem 1 to answer the problem posed by Ossona de Mendez and Nešetřil in [23]:

Problem. Is there a simple linear time algorithm to check $t d(G) \leqslant t$ for fixed $t$ ? Is there a simple linear time algorithm to compute a rooted forest $Y$ of height $t$ such that $G \subseteq \operatorname{clos}(Y)$ (provided that such a rooted forest exists)?

The problem is motivated by the fact that treedepth-being a minorclosed property - can be expressed in monadic second order logic and thus one can employ Courcelle's theorem [8] to compute the treedepth of a graph of bounded treewidth in linear time. The above problem is motivated by the fact that the running time of this approach is unclear. More specifically, the standard proof of Courcelle's involves creating a tree-automaton whose size cannot be bounded by any elementary function in the formula size unless $\mathrm{P}=\mathrm{NP}$ [16]. Here we will show that we can use the algorithm presented in Chapter 3 to give a much more direct and simpler algorithm.

Proposition 2 ([23]). Let $G$ be a graph of treedepth $t$. Then a treedepth decomposition which is the tree given by a depth first search of $G$ is a $2^{t}$ approximation of the treedepth.

It is easy to see that the tree given by a depth first search of a graph gives a treedepth decomposition of the graph. As a path of length $2^{t}$ would witness the fact that the treedepth is larger than $t$, one either obtains a DFS of height most $2^{t}$ or can correctly conclude that the given graph has treedepth $>t$. 
Proposition 3 ([23]). Let $G$ be a graph and let $T$ be DFS-tree of $G$. Then there exists a path decomposition of $G$ whose width is the height of $T$. This path decomposition can be computed in linear time.

The algorithm we presented in Chapter 3 expected a tree decomposition as part of its input. Thanks to the above two lemmas we can construct an algorithm which only takes a graph as its input, cf. Algorithm 3 .

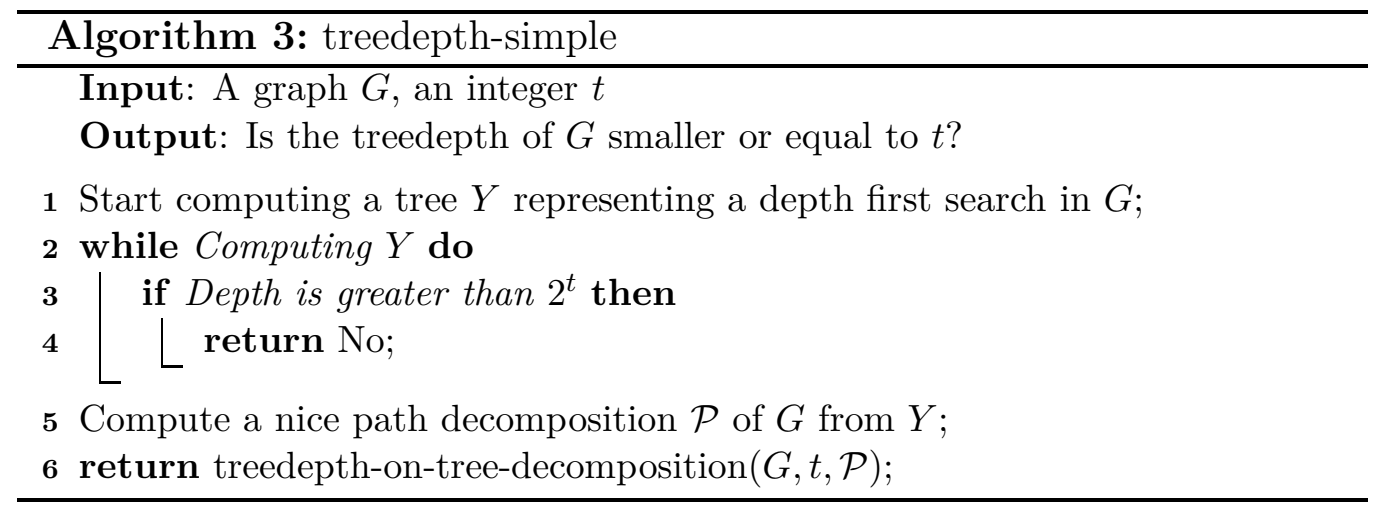

The following theorem now follows from Theorem 1 and Algorithm 3 ,

Theorem 2. There is a simple algorithm to decide whether the treedepth of a graph is at most $t$ in time and space $2^{2^{O(t)}} n$ and, in the positive case, output a treedepth decomposition witnessing this fact.

We point out that Algorithm 3 can be made to run in logarithmic space. Lemma 18. The algorithm in Algorithm 3 can be made to run in logarithmic space for a fixed treedepth $t$.

Proof. Sketch: It is easy to see that a depth first search can be implemented in such a way that only the current path from the root of the search tree to the leaf must be kept in memory. Since the depth of our search is bounded by the constant $2^{t}$ we can compute the search tree in logarithmic space. The contents of each bag in the path decomposition are precisely the paths from the root to a leaf of the search tree. This means that we can compute the bags of the path decomposition in parallel to the computation of the search tree. Since for path decompositions we only need the forget and introduce operations, we only need to keep two bags in memory at any point. Since the size of the tables is bounded by a function in $t$, it follows that the algorithm in 
Algorithm 3 can be implemented in such a way that it only uses logarithmic space.

All in all, we consider our algorithm to solve the stated problem.

\section{Fast Algorithm}

We can also extend the algorithm in Chapter 3 to get a fast algorithm. The simple version we presented in the previous chapter might be simple, but it runs in double exponential time. This is because to get a tree decomposition 3 whose width is bounded in $t$ we use a depth first search tree, which is very easy to use and does not require any complex mathematical tools, but only gives us a tree decompositions whose width is $2^{t}$. If we could bound the width of the tree decomposition we compute before running Algorithm 1 linearly in $t$ then we could get a much better running time. We want to show this is actually easily possible using known results.

First notice that $t w(G) \leqslant p w(G) \leqslant t d(G)$ for any graph $G$. This fact was implicitly proven in Chapter 4 since we showed how it easy to construct a path decomposition of width $t$ from a treedepth decomposition of height $t$. It follows then that if we bound the width of the tree decomposition linearly on the treewidth of the graph we will also be bounding it on the treedepth of graph. There is a recent results which proofs there exists an algorithm which runs in time $2^{O(t)} n$ and calculates a 5 -approximation tree decomposition for a graph [5]. The algorithm in Algorithm 4 shows an algorithm that uses these two facts to give a fast algorithm.

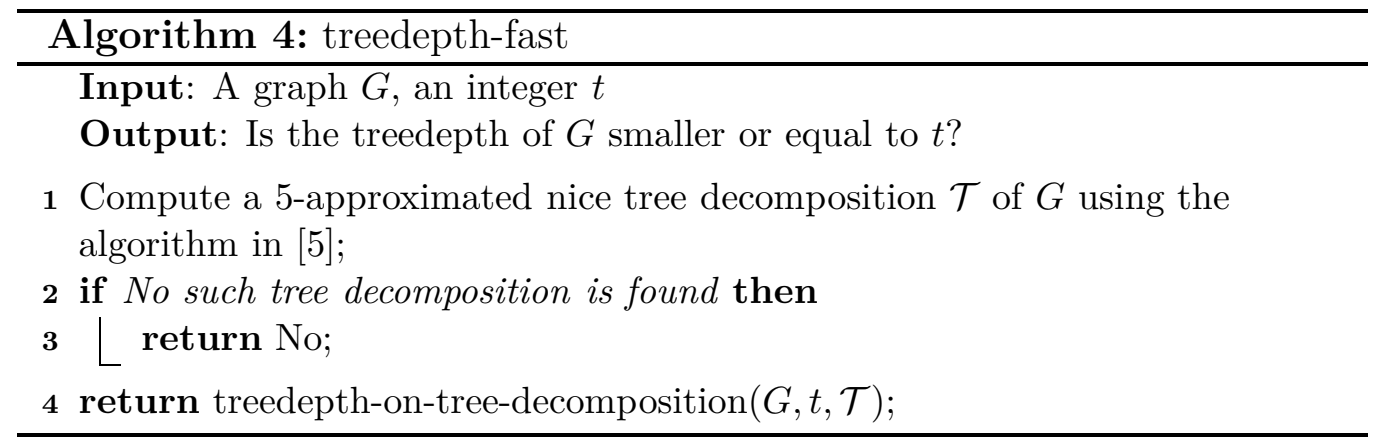

\footnotetext{
${ }^{3}$ actually a path decomposition
} 
Lemma 19. Algorithm 4 decides the treedepth of the input graph $G$.

Proof. Since $t w(G) \leqslant t d(G)$ if the graph $G$ has treedepth $t$ then there must exist a tree decomposition of width at most $5 t$ which is a 5 -approximation for the treewidth of the graph. Thus returning with a negative result on line 3 is correct. From Theorem 1 we know that the call to Algorithm 1 decides if $G$ has treedepth $t$, thus the lemma follows.

Lemma 20. Algorithm 4 runs in time $2^{O\left(t^{2}\right)}$ on the input graph $G$ and integer $t$.

Proof. Since $t w(G) \leqslant t d(G)$ of follows that the width of the tree decomposition $\mathcal{T}$ is at most $5 t$. From [5] we know that the running time of computing the tree decomposition is $2^{O(t)} n$ and from Theorem 1 the the call to Algorithm 1 is $2^{O(t w)} n$, where $w$ is the width of $\mathcal{T}$. Since $w \leqslant 5 t$ it follows that the running time of the call to Algorithm 1 is $2^{O(t \cdot 5 t)} \cdot n=2^{O\left(t^{2}\right)} \cdot n$.

For the same reason as in previous chapter, backtracking can also be used in this algorithm to compute an actual treedepth decomposition of height $t$ or less. Thus the following theorem follows.

Theorem 3. Let $G$ be a graph of size $n$. Deciding if $G$ has a treedepth decomposition of height $t$ and constructing such a treedepth decomposition can be computed in time $2^{O\left(t^{2}\right)} n$.

To the best of our knowledge the best running time to compute the exact treewidth $w$ of a graph takes time $2^{O\left(w^{3}\right)} n$, which means that our algorithm computes treedepth of a graph faster than the best known algorithm for treewidth computes the treewidth of a graph.

\section{Treedepth and Chordal graphs}

As mentioned in the introduction, deciding treedepth remains NP-hard even on chordal graphs. Interestingly, the special structure of tree decompositions of chordal graphs can be used to reduce the running time of our algorithm significantly with only minor changes. To the best of our knowledge, no such algorithm was known so far (an algorithm with exponential dependence on the number of cliques in a chordal graph was presented in [1]). Since obtaining an optimal tree decomposition for chordal graphs is possible in linear time, we do not need the treewidth approximation here. 
Lemma 21. Given a chordal graph $G$ and an integer $t$, one can decide in time and space $2^{O(t \log t)} \cdot n$ whether $\operatorname{td}(G) \leqslant t$ and in the positive case output a treedepth decomposition of that height.

Proof. Since adding a universal vertex to a chordal graph does not violate the chordality, we tacitly assume in the following that such a vertex $r$ exists. First, check whether $\omega(G)>t$ and if that is the case, output that the treedepth of $G$ is greater than $t$. Otherwise, $\omega(G) \leqslant t$ which implies that $\mathbf{t w}(G) \leqslant t$. Compute a clique tree of $G$ in linear time (cf. [2]), i.e. a tree decomposition of $G$ in which every bag induces a clique.

If we now run Algorithm 2 on $G$ we can show that only partial decompositions whose forest is a path are kept during each step of the dynamic programming: consider a bag $X$ and a set of restrictions $R$ computed by the algorithm. For any $(F, X, h) \in R$, the condition $E(G[X]) \subseteq E(\operatorname{clos}(F)[X])$ must be fulfilled (in the join- and introduce-case this is explicitly enforced and it is easy to see that the forget-case cannot create a non-path from a path). Therefore, all elements of $X$ lie in a single path from the root to a leaf in $F$-but since in a restriction every leaf of $F$ must be a member of $X$, this path is exactly $F$. The maximum number of restrictions of height at most $t$ and whose forest is a single path is bounded by $2^{O(t \log t)}$, cf. proof of Lemma 15. If we modify the introduce- and join-procedure of Algorithm 2 to only generate restrictions whose forests are paths, which by the previous observation are the only restrictions that would be kept in any case, the running time reduces to the claimed bound.

\section{Conclusions and Further Research}

We provide an explicit simple self contained algorithm, i.e. an algorithm which does not rely on any other complex results, which for a fixed $t$ decides if a graph $G$ has treedepth $t$ or computes a treedepth decomposition of height $t$ if one exists in linear time. This answers an open question posed in [23]. We also provide an explicit algorithm to decide the treedepth or construct a minimal treedepth decomposition of a given graph in time $2^{O\left(t^{2}\right)} n$.

A natural question that arises is whether one can find a constant-factor approximation for treedepth in single-exponential time, similar to the algorithm for treewidth. Such an algorithm would be interesting in the sense that it would remove the dependency of the algorithm provided in this paper 
from the treewidth-approximation (hoping that a direct approximation of treedepth would be simpler).

On the topic of width-measures, it still remains open whether graphs of low treedepth admit fast algorithms that are impossible on graphs of low pathwidth. This is motivated further by the fact that the construction proving lower bounds on graphs of bounded pathwidth clearly contain very long paths and thus have high treedepth [21]. Proving similar bounds for graphs of bounded treedepth would be equally insightful.

\section{References}

[1] B. Aspvall and P. Heggernes. Finding minimum height elimination trees for interval graphs in polynomial time. BIT Numerical Mathematics, 34(4):484-509, 1994.

[2] J. R. S. Blair and B. Peyton. An introduction to chordal graphs and clique trees. In Graph theory and sparse matrix computation, pages 1-29. Springer, 1993.

[3] H. L. Bodlaender. A tourist guide through treewidth. Acta Cybernetica, 11:1-21, 1993.

[4] H. L. Bodlaender, J. S. Deogun, K. Jansen, T. Kloks, D. Kratsch, H. Müller, and Z. Tuza. Rankings of graphs. SIAM Journal of Discrete Mathematics, 11(1):168-181, 1998.

[5] H. L. Bodlaender, P. G. Drange, M. S. Dregi, F. V. Fomin, D. Lokshtanov, and M. Pilipczuk. A $O\left(c^{k} n\right)$ 5-approximation algorithm for treewidth. CoRR, abs/1304.6321, 2013.

[6] H. L. Bodlaender, J. R. Gilbert, H. Hafsteinsson, and T. Kloks. Approximating treewidth, pathwidth, frontsize, and shortest elimination tree. Journal of Algorithms, 18(2):238-255, 1995.

[7] H. L. Bodlaender and D. Kratsch. Personal communication, 2014.

[8] B. Courcelle. The Monadic Second-Order Theory of Graphs. I. Recognizable Sets of Finite graphs. Information and Computation, 85:12-75, 1990. 
[9] J. S. Deogun, T. Kloks, D. Kratsch, and H. Müller. On vertex ranking for permutations and other graphs. In $S T A C S$, number 775 in LNCS, pages 747-758. Springer, 1994.

[10] D. Dereniowski and A. Nadolski. Vertex rankings of chordal graphs and weighted trees. Information Processing Letters, 98, 2006.

[11] R. Diestel. Graph Theory. Springer-Verlag, Heidelberg, 4th edition, 2010.

[12] R. G. Downey and M. R. Fellows. Parameterized Complexity. SpringerVerlag, 1999.

[13] I. S. Duff and J. K. Reid. The multifrontal solution of indefinite sparse symmetric linear equations. ACM Transactions on Mathematical Software, 9(3):302-325, 1983.

[14] J. Flum and M. Grohe. Parameterized Complexity Theory. SpringerVerlag, 2006.

[15] F. V. Fomin, A. C. Giannopoulou, and M. Pilipczuk. Computing treedepth faster than $2^{n}$. In Parameterized and Exact Computation, volume 8246 of Lecture Notes in Computer Science, pages 137-149. Springer International Publishing, 2013.

[16] M. Frick and M. Grohe. The complexity of first-order and monadic second-order logic revisited. Annals of Pure and Applied Logic, 130(1$3): 3-31,2004$.

[17] M. Katchalski, W. McCuaig, and S. Seager. Ordered colourings. Discrete Mathematics, 142(1-3):141-154, 1995.

[18] K. Kaya and B. Uçar. Constructing elimination trees for sparse unsymmetric matrices. SIAM Journal on Matrix Analysis and Applications, $34(2): 345-354,2013$.

[19] C. E. Leiserson. Area-efficient graph layouts (for VLSI). In FOCS, pages 270-281, 1980.

[20] J. W. H. Liu. The role of elimination trees in sparse factorization. SIAM Journal on Matrix Analysis and Applications, 11(1):134-172, 1990. 
[21] D. Lokshtanov, D. Marx, and S. Saurabh. Known algorithms on graphs on bounded treewidth are probably optimal. In D. Randall, editor, Proc. of 22nd SODA, pages 777-789. SIAM, 2011.

[22] J. Nešetřil and P. Ossona de Mendez. Grad and classes with bounded expansion I. Decompositions. European Journal of Combinatorics, 29(3):760-776, 2008.

[23] J. Nešetřil and P. Ossona de Mendez. Sparsity: Graphs, Structures, and Algorithms, volume 28 of Algorithms and Combinatorics. Springer, 2012.

[24] R. Niedermeier. Invitation to Fixed-Parameter Algorithms. Oxford University Press, 2006.

[25] A. Pothen. The complexity of optimal elimination trees. Technical Report CS-88-13, Pennsylvannia State University, 1988.

[26] A. Pothen, H. D. Simon, and K.-P. Liou. Partitioning sparse matrices with eigenvectors of graphs. SIAM Journal of Matrix Analysis and Applications, 11(3):430-452, 1990.

[27] N. Robertson and P. D. Seymour. Graph minors XIII. The disjoint paths problem. Journal of Combinatorial Theory, Series B, 63:65-110, 1995.

[28] P. Scheffler. Die Baumweite von Graphen als ein Maß für die Kompliziertheit algorithmischer Probleme. PhD thesis, Akademie der Wissenschaften der DDR, Berlin, Germany, 1989.

[29] D. A. Spielman and S.-H. Teng. Spectral partitioning works: Planar graphs and finite element meshes. In FOCS, pages 96-105, 1996. 